\title{
On Radicals of Semirings and Related Problems
}

\author{
Y. Katsov \\ Department of Mathematics and Computer Science \\ Hanover College, Hanover, IN 47243-0890, USA \\ katsov@hanover.edu \\ T. G. Nam \\ Department of Mathematics \\ Dong Thap Pedagogical University, Dong Thap, Vietnam \\ trangiangnam05@yahoo.com
}

\begin{abstract}
The aim of this paper is to develop an 'external' Kurosh-Amitsur radical theory of semirings and, using this approach, to obtain some fundamental results regarding two Jacobson type of radicals - the Jacobson-Bourne, $J$-, radical and a very natural its variation, $J_{s^{-}}$ radical - of hemirings, as well as the Brown-McCoy, $\mathcal{R}_{B M^{-}}$, radical of hemirings. Among the new central results of the paper, we single out the following ones: Theorems unifying two, internal and external, approches to the Kurosh-Amitzur radical theory of hemirings; A characterization of $J$-semisimple hemirings; A description of $J$-semisimple congruence-simple hemirings; A characterization of finite additively-idempotent $J_{s}$-semisimple hemirings; Complete discriptions of $\mathcal{R}_{B M}$-semisimple commutative and latticeordered hemirings; Semiring versions of the well-known classical ring results - Nakayama's and Hopkins Lemmas and Jacobson-Chevalley Density Theorem; Establishing the fundamental relationship between the radicals $J, J_{s}$, and $\mathcal{R}_{B M}$ of hemirings $R$ and matrix hemirings $M_{n}(R)$; Establishing the matric-extensibleness (see, e.g., [4, Section 4.9]) of the radical classes of the Jacobson, Brown-McCoy, and $J_{s^{-}}$, radicals of hemirings; Showing that the $J$-semisimplicity, $J_{s}$-semisimplicity, and $\mathcal{R}_{B M}$-semisimplicity of semirings are Morita invariant properties.
\end{abstract}


2010 Mathematics Subject Classifications: Primary 16Y60, 16D99, 16N99; Secondary 18A40, 18G05, 12K10

Key words: radical classes of semirings, semisimple classes of semirings, Jacobson radicals of semirings, Brown-McCoy radicals of semirings, congruence-simple semirings, irreducible semimodules, Morita equivalence of semirings.

\section{Introduction}

As is well known, radical theory and radicals of algebraic structures, originated in the beginning of the last century by Wedderburn and Köthe for rings (see, e.g., [4]), constitute important "classical" areas of the sustained interest in algebraic research which very often also initiate research in new directions in other branches of mathematics. In particular, studying of some analogs of the classical, Jacobson and Brown-McCoy, ring radicals in a semiring setting commenced in 1950-60s in [3, [9], [19], and [20]. As well, a Kurosh-Amitzur radical theory in a semiring setting has been started in [26], [27], [28], [29], and [30] (see, also, [31] and [32]), and then has been significantly advanced in [6], 25], [7], and [8]. Motivated by the Kurosh-Amitzur radical theory for rings (see, e.g., [4]), the authors of the latter papers have developed an 'internal' Kurosh-Amitsur radical theory for semirings, i.e., the radical theory build "axiomatically" exclusively within the class $\mathbb{H}$ of all hemirings (semirings not necessarily possessing units) and not involving representations of hemirings, that is, semimodules over them. Although the main concepts of the both radical theories - for rings and hemirings - are defined quite similarly, there are considerable differences between these theories as well as all considerations and proofs for semirings, not surprisingly, are significantly more complicated and demand innovative ideas and techniques. As an algebraic objects, semirings certainly are the most natural generalization of such algebraic systems as rings and bounded distributive lattices, and investigating semirings and their representations, one should undoubtedly use methods and techniques of both ring and lattice theory as well as diverse techniques and methods of categorical and universal algebra. Thus, a wide variety of the algebraic techniques used in studying semirings and their representations/semimodules perhaps explains why research on a Kurosh-Amitsur radical theory for semirings is still behind of that for rings.

The principal task here is to initiate an 'external' Kurosh-Amitsur radical theory for semirings - a radical theory based, at this time, on representations, semimodules, of semirings - in the spirit of, and by analogy with, the external radical theory for rings that can be found, for example, in [4, Section 3.14]; and then, based on it, to present a series of fundamental 
results regarding the Jacobson and Brown-McCoy radicals of hemirings, as well as to answer to some questions left open in the earlier publications, mentioned above, on these topics.

The paper is organized as follows. In Section 2, for the reader's convenience divided into two subsections, we included all subsequently necessary notions and facts on semirings and semimodules, as well as on an internal Kurosh-Amitzur radical theory of semirings.

In Section 3, after developing an external Kurosh-Amitsur radical theory of semirings, we illustrate this approach by considering in more details two Jacobson type of radicals - the Jacobson-Bourne, $J_{-}$, radical and a very natural its variation, $J_{s}$-radical - of hemirings, which coincide for rings but are different in general, as well as the Brown-McCoy, $\mathcal{R}_{B M^{-}}$, radical of hemirings. Among the new results of this section, which are also among the central results of the paper, we single out the following ones: Theorems 3.2, 3.3 , and 3.4, actually unifying two, internal and external, approaches to the Kurosh-Amitzur radical theory of hemirings; Corollary 3.8, characterizing $J$-semisimple hemirings and answering a question left open in [19, Theorem 3.3 , p. 12]; a description of $J$-semisimple congruence-simple hemirings (Theorem 3.10); a characterization of finite additively-idempotent $J_{s}$-semisimple hemirings (Theorem 3.11); complete descriptions of $\mathcal{R}_{B M}$-semisimple commutative and lattice-ordered hemirings (Corollaries 3.16 and 3.17).

In Section 4, reducing our semiring settings to corresponding ring ones, we establish semiring versions of the well-known classical ring resultsNakayama's and Hopkins Lemmas and Jacobson-Chevalley Density Theorem (Theorem 4.3, Corollary 4.4, and Theorem 4.5, respectively), which are among the main results of the paper.

Section 5 among other, in our view interesting and useful, observations, contains the following main results of the paper: Theorem 5.8 and Corollary 5.11, establishing the fundamental relationship between the radicals $J, J_{s}$, and $\mathcal{R}_{B M}$ of hemirings $R$ and matrix hemirings $M_{n}(R), n \geq 1$, and particularly extending [3, Theorem 9] from semirings to general hemirings; Theorem 5.14, establishing the matric-extensibleness (see, e.g., [4, Section $4.9]$ ) of the radical classes of the Jacobson, Brown-McCoy, and $J_{s^{-}}$, radicals of hemirings; and Theorem 5.17, showing that the $J$-semisimplicity, $J_{s^{-}}$ semisimplicity, and $\mathcal{R}_{B M}$-semisimplicity of semirings are Morita invariant properties.

Finally, in the course of the paper, there have been stated several, in our view interesting and promising, problems; also, all notions and facts of categorical algebra, used here without any comments, can be found in [22], and for notions and facts from semiring theory we refer to [5]. 


\section{Basic Concepts}

\subsection{Preliminaries on Semirings}

Recall [5] that a hemiring is an algebra $(R,+, \cdot, 0)$ such that the following conditions are satisfied:

(1) $(R,+, 0)$ is a commutative monoid with identity element 0 ;

(2) $(R, \cdot)$ is a semigroup;

(3) Multiplication distributes over addition from either side;

(4) $0 r=0=r 0$ for all $r \in R$.

And a hemiring $R$ is a semiring if its multiplicative $\operatorname{semigroup}(R, \cdot)$ actually is a monoid $(R, \cdot, 1)$ with identity element 1 . Any hemiring $R$ can be naturally considered as an ideal of its Dorroh extension by $\mathbb{N}:=$ $\{0,1, \ldots\}$ [5, p.3], $R^{1}:=R \times \mathbb{N}$, which is a semiring with the identity element $(0,1) \in R^{1}$ and operations of addition and multiplication defined by $(r, n)+(s, m):=(r+s, n+m)$ and $(r, n)(s, m):=(r s+m r+n s, n m)$ for all $(r, n),(s, m) \in R^{1}$.

A hemiring $R$ is additively cancellative if $a+c=b+c$ implies $a=b$ for all $a, b, c \in R$. A nonempty subset $I$ of a hemiring $R$ is subtractive if, for all $x, a \in R$, from $x+a, a \in I$ follows that $x \in I$, too. The subtractive closure $\bar{I}$ of an ideal $I$ of a hemiring $R$ is the smallest subtractive ideal of $R$ containing $I$, and it is obviously defined as $\bar{I}:=\{r \in R \mid r+x \in I$ for some $x \in I\}$; also, it is clear that an ideal $I$ a hemiring $R$ is subtractive iff it coincides with its subtractive closure, i.e., $I=\bar{I}$. Clearly, 0 and $R$ are subtractive ideals for each hemiring $R$. By $\mathcal{I}(R)$ and $\mathcal{S I}(R)$ are denoted the sets of all ideals and all subtractive ideals of a hemiring $R$, respectively; and a hemiring $R$ is called subtractive if $\mathcal{I}(R)=\mathcal{S I}(R)$.

A hemiring $R$ is lattice-ordered [5, Section 21] iff it is also a lattice $(R, \vee, \wedge)$, and for all $a, b \in R$, the following conditions are satisfied: $a+b=$ $a \vee b$ and, with respect to the partial order naturally induced by the lattice operations, $a b \leq a \wedge b$.

As for rings, for any homomorphism $f: R \longrightarrow S$ between hemirings $R$ and $S$, there exists a subtractive ideal, the kernel, $\operatorname{Ker}(f):=\{a \mid f(a)=$ $0\} \subseteq R$ of $f$. A surjective hemiring homomorphism $f: R \longrightarrow S$ is a semiisomorphism if $\operatorname{Ker}(f)=0$. As usual, the direct product $R=\prod_{i \in I} R_{i}$ of a family $\left(R_{i}\right)_{i \in I}$ of hemirings $R_{i}$ consists of the elements $r=\left(r_{i}\right)_{i \in I}$ for $s_{i} \in R_{i}$ and is determined by the surjective homomorphisms $\pi_{i}: R \longrightarrow R_{i}$ defined by $\pi_{i}(r)=r_{i}$; and a subhemiring $S$ of $R$ is called a subdirect product $S=\prod_{i \in I}^{s u b} R_{i}$ of $\left(R_{i}\right)_{i \in I}$ if, for each $\pi_{i}$, the restriction $\left.\pi_{i}\right|_{S}: S \longrightarrow R_{i}$ is also surjective (see, e.g., [8, p. 194]). 
Any ideal $I$ of a hemiring $R$ induces a congruence relation $\equiv_{I}$ on $R$, called the Bourne relation [5, p.78], given by $r \equiv_{I} r^{\prime}$ iff there exist elements $x, x^{\prime} \in I$ such that $r+x=r^{\prime}+x^{\prime}$; and $R / I$ denotes the factor hemiring $R / \equiv_{I}$. Also, it is easy to see that the congruences $\equiv_{I}$ and $\equiv_{\bar{I}}$ on $R$ coincide for any ideal $I$ of $R$, and hence, $R / I=R / \bar{I}$ hold for all ideals $I$ of $R$.

As usual, a left $R$-semimodule over a hemiring $R$ is a commutative monoid $\left(M,+, 0_{M}\right)$ together with a scalar multiplication $(r, m) \mapsto r m$ from $R \times M$ to $M$ which satisfies the following identities for all $r, r^{\prime} \in R$ and $m, m^{\prime} \in M:$

(1) $\left(r r^{\prime}\right) m=r\left(r^{\prime} m\right)$;

(2) $r\left(m+m^{\prime}\right)=r m+r m^{\prime}$;

(3) $\left(r+r^{\prime}\right) m=r m+r^{\prime} m$;

(4) $r 0_{M}=0_{M}=0 \mathrm{~m}$.

Right semimodules over a hemiring $R$ and homomorphisms between semimodules are defined in the standard manner. If a hemiring $R$ is a semiring, then all $R$-semimodules over $R$ are unitary ones. And, from now on, let $\mathcal{M}_{R}$ and ${ }_{R} \mathcal{M}$ denote the categories of right and left semimodules, respectively, over a semiring $R$. As usual (see, for example, [5, Chapter $17]$ ), in the category ${ }_{R} \mathcal{M}$ of left semimodules over a semiring $R$, a free (left) semimodule with a basis set $I$ is a direct sum (a coproduct) $\sum_{i \in I} R_{i}, R_{i} \cong$ ${ }_{R} R, i \in I$, of $I$ copies of ${ }_{R} R$; and a projective left semimodule in ${ }_{R} \mathcal{M}$ is just a retract of a free semimodule. A semimodule ${ }_{R} M$ is finitely generated iff it is a homomorphic image of a free semimodule with a finite basis set. A left semimodule $M$ over a hemiring $R$ is cancellative if $x+z=y+z$ implies $x=y$ for all $x, y, z \in M$. A nonempty subset $N$ of an $R$-semimodule $M$ is subtractive if, for all $x, y \in M$, from $x+y, x \in N$ follows that $y \in N$, too.

The usual concepts of the Descending Chain Condition and artinian modules of theory of modules over rings, as well as results involving them, are easily extended in an obvious fashion (see, e.g., [13]) to a context of semimodules over semirings. As for the modules over rings, $(0: M)$ or $(0: M)_{R}$ denotes the annihilator of a left $R$-semimodule ${ }_{R} M$, i.e., $(0$ : $M)_{R}:=\{r \in R \mid r M=0\}$; and ${ }_{R} M$ is faithful iff $(0: M)_{R}=0$. Finally and similarly to the case of module over rings, the following observations can be easily verified and will prove to be useful in sequel:

Proposition 2.1. (cf. [4, Proposition 3.14.1]) Let $R$ be a hemiring, and $I$ $\in \mathcal{I}(R)$.

(i) If $M$ is a left $R / I$-semimodule, then under the scalar multiplication

$$
r m=\bar{r} m,
$$

$M$ becomes a left $R$-semimodule with $I \subseteq(0: M)_{R}$. 
(ii) If $M$ is a left $R$-semimodule with $I \subseteq(0: M)_{R}$, then $M$ is a left $R / I$-semimodule under the scalar multiplication

$$
\bar{r} m=r m \text {. }
$$

(iii) Every subsemimodule of the left $R / I$-semimodule $M$ is a subsemimodule of the left $R$-semimodule $M$, and the converse is also true when $I \subseteq(0: M)_{R}$.

(iv) $(0: M)_{R / I}=(0: M)_{R} / I$.

Proof. (i), (ii) and (iii) are clear.

(iv) Obliviously, $(0: M)_{R / I} \supseteq(0: M)_{R} / I$. Conversely, for any $\bar{r} \in(0$ : $M)_{R / I}, \bar{r} m=0$ for all $m \in M$, and, hence, $r m=0$ for all $m \in M$. Thus $r \in(0: M)_{R}$.

\subsection{Preliminaries on Radical Theory of Semirings}

In this subsection, we briefly sketch the basic concepts of an 'internal' Kurosh-Amitsur radical theory of semirings - in other words, the KuroshAmitsur radical theory build exclusively within the class $\mathbb{H}$ of all hemirings without using representations of hemirings - originated by D. M. Olson and his coauthors in a series of papers [26]-29], and then considerably advanced in [6], [25], [7, and [8]. As was shown in the latter papers (see, for instance, [25]), similarly to the radical theory of rings (see, e.g., [4]), there are three equivalent approaches to the Kurosh-Amitsur radical theory of semiringsby means of radical classes, radical operators, and semisimple classes, independently defined in a fixed universal class $\mathbb{U} \subseteq \mathbb{H}$ of hemirings. Herewith, a nonempty subclass $\mathbb{U}$ of $\mathbb{H}$ is called universal if $\mathbb{U}$ is a hereditary $(R \in \mathbb{U}$ implies $\mathcal{I}(R) \subseteq \mathbb{U})$ and homomorphically closed $(R \in \mathbb{U}$ implies $\varphi(R) \in \mathbb{U}$ for every hemiring homomorphism $\varphi$ ) subclass in $\mathbb{H}$. Furthermore, by [25, Definition 3.1], a nonempty subclass $\mathbb{R}$ of a fixed universal class $\mathbb{U} \subseteq \mathbb{H}$ is called a radical class of $\mathbb{U}$ if $\mathbb{R}$ satisfies the following two conditions: (i) $\mathbb{R}$ is homomorphically closed; (ii) For every hemiring $R \in \mathbb{U} \backslash \mathbb{R}$, there exists a subtractive ideal $I \in \mathcal{S I}(R) \backslash\{R\}$ such that $\mathcal{I}(R / I) \cap \mathbb{R}=\{(0)\}$, where $(0)$ is the trivial, zero, hemiring.

Analogously to the case of rings (see, e.g., [4, Chapter II]), there have been established the following characterizations of radical classes for hemirings:

Theorem 2.2. ([25, Theorems 3.2 and 4.7]) For a subclass $\mathbb{R}$ of a universal class $\mathbb{U}$, the following conditions are equivalent:

(1) $\mathbb{R}$ is a radical class of $\mathbb{U}$.

(2) $\mathbb{R}$ satisfies the following two properties: 
(i) If $R \in \mathbb{R}$, then for every nonzero surjective hemiring homomorphism $R \longrightarrow S$ there exists a nonzero ideal $I$ of $S$ such that $I \in \mathbb{R}$;

(ii) If $R \in \mathbb{U}$ and for every nonzero surjective hemiring homomorphism $R \longrightarrow S$ there exists a nonzero ideal $I$ of $S$ such that $I \in \mathbb{R}$, then $R \in \mathbb{R}$.

(3) The following three properties are true for $\mathbb{R}$ :

(i) $\mathbb{R}$ is homomorphically closed;

(ii) $\mathbb{R}$ is extensionally closed in $\mathbb{U}$ - for all $R \in \mathbb{U}$ and $I \in \mathcal{I}(R)$, if $I \in \mathbb{R}$ and $R / I \in \mathbb{R}$, then $R \in \mathbb{R}$;

(iii) $\mathbb{R}$ has the inductive property - for any $R \in \mathbb{U}$ and ascending chain of ideals $I_{1} \subseteq \ldots \subseteq I_{\lambda} \subseteq$...with all $I_{\lambda} \in \mathcal{I}(R) \cap \mathbb{R}$, the ideal $\cup I_{\lambda} \in \mathbb{R}$, too.

As was shown in [25, Theorem 5.3] (and for a more restricted class of universal classes in [27, p. 309 and Theorem 17], too), the general method of a construction of the upper radical $\mathcal{U} \mathbb{S}$ for a regular class of rings $\mathbb{S}$ (see, e.g., [4, Theorem 2.2..3]) can be extended to a hemiring setting as well. Recall (see [25, p. 542 and Theorem 5.3] or [7, p. 182]) that a subclass $\mathbb{S}$ of a universal class $\mathbb{U} \subseteq \mathbb{H}$ is a regular class if, for any nonzero ideal $I$ of a hemiring $R \in \mathbb{S}$, there exists a nonzero surjective hemiring homomorphism $I \longrightarrow S$ with $S \in \mathbb{S}$. Since Theorem 5.3 of [25] was proved under the assumption that a regular subclass $\mathbb{S}$ of a universal class $\mathbb{U}$ is an isomorphically closed class, we have found to be reasonable to propose here an alternative, new proof of it eliminating this assumption.

Theorem 2.3. ( $c f$. [25, Theorem 5.3]) If $\mathbb{S}$ is a regular subclass of a universal class $\mathbb{U} \subseteq \mathbb{H}$, then the class

$$
\mathcal{U} \mathbb{S}=\{R \in \mathbb{U} \mid \text { Rhas no nonzero homomorphic image in } \mathbb{S}\}
$$

is a radical class of $\mathbb{U}$ and $\mathbb{S} \cap \mathbb{U S}=\{(0)\}$; moreover, $\mathcal{U} \mathbb{S}$ is the largest radical class in $\mathbb{U}$ having zero intersection with $\mathbb{S}$.

Proof. It is obvious, as in the proof of [25, Theorem 5.3], that $\mathcal{U} \mathbb{S}$ is homomorphically closed.

Let us show that $\mathcal{U} \mathbb{S}$ is extensionally closed. Suppose that for a hemiring $R$ and and ideal $I \in \mathcal{I}(R)$ we have that $I, R / I \in \mathcal{U} \mathbb{S}$, but $R \notin \mathcal{U} \mathbb{S}$. Then, there exists a nonzero surjective homomorphism $f: R \longrightarrow S$ with $S \in \mathbb{S}$, and consider two possible cases:

a) $I \nsubseteq \operatorname{Ker}(f)$. Then, for $f(I)$ is a nonzero ideal of $S \in \mathbb{S}$ and $\mathbb{S}$ is a regular class, there is a nonzero surjective homomorphism $g: f(I) \longrightarrow H$ with $H \in \mathbb{S}$; hence, $\left.g f\right|_{I}: I \longrightarrow H$, where $\left.f\right|_{I}$ is the restriction of $f$ on $I$, 
is a nonzero surjective homomorphism. Therefore, one gets a contradiction $I \notin \mathcal{U} \mathbb{S}$.

b) $I \subseteq \operatorname{Ker}(f)$. Then, for the mapping $h: R / I \longrightarrow S$, defined by $h(\bar{r})=f(r)$, is a nonzero surjective homomorphism, one has a contradiction $R / I \notin \mathcal{U} \mathbb{S}$.

Let us show that $\mathcal{U} \mathbb{S}$ has the inductive property. Suppose that for $R \in$ $\mathbb{U}$, a chain $I_{1} \subseteq \ldots \subseteq I_{\lambda} \subseteq \ldots$ with all $I_{\lambda} \in \mathcal{I}(R) \cap \mathcal{U} \mathbb{S}$, and $I=\cup I_{\lambda}$, we have $I \notin \mathcal{U} \mathbb{S}$. Then, there exists a nonzero surjective homomorphism $f: I \longrightarrow J$ with $J \in \mathbb{S}$. Hence, there exists an element $a_{\lambda} \in I_{\lambda} \subseteq I$ such that $f\left(a_{\lambda}\right) \neq 0$, and $\left.f\right|_{I_{\lambda}}\left(I_{\lambda}\right)$ is a nonzero ideal of $J \in \mathbb{S}$. Since $\mathbb{S}$ is a regular class, there exist nonzero surjective homomorphisms $g:\left.f\right|_{I_{\lambda}}\left(I_{\lambda}\right) \longrightarrow K \in \mathbb{S}$ and $\left.g f\right|_{I_{\lambda}}: I_{\lambda} \longrightarrow K \in \mathbb{S}$, and, therefore, we have a contradiction $I_{\lambda} \notin \mathcal{U} \mathbb{S}$.

By Theorem $2.2(3)$, we have established that $\mathcal{U} \mathbb{S}$ is a radical class of $\mathbb{U}$.

The rest is almost obvious and can be shown as in [25, Theorem 5.3].

Following [7, Definition 2.4] (or [25, Definition 4.1]), a mapping $\varrho: \mathbb{U} \longrightarrow$ $\mathbb{U}$ is called a radical operator in $\mathbb{U}$ if each hemiring $R \in \mathbb{U}$ has an image $\varrho(R) \in \mathcal{S I}(R) \subseteq \mathbb{U}$ such that the following conditions are satisfied for all $R, S \in \mathbb{U}:$

(i) $\varphi(\varrho(R)) \subseteq \varrho(\varphi(R))$ for each homomorphism $\varphi: R \longrightarrow S$;

(ii) $\varrho(R / \varrho(R))=(0)$;

(iii) For every nonzero ideal $I$ of $R, \varrho(I)=I$ implies that $I \subseteq \varrho(R)$;

(iv) $\varrho(\varrho(R))=\varrho(R)$.

Terming an ideal $I \in \mathcal{I}(R) \cap \mathbb{R}$ of a hemiring $R$ for a radical class $\mathbb{R}$ an $\mathbb{R}$-ideal of $R$, it was shown [25, Theorem 3.7] that all $\mathbb{R}$-ideals of $R \in \mathbb{U}$ are contained in the greatest ideal of this kind, called the $\mathbb{R}$-radical of $R$. More precisely, we have the following fact:

Theorem 2.4. ([7, Theorem 2.3]) For a radical class $\mathbb{R}$ of $\mathbb{U}$ and $R \in \mathbb{U}$, the union $\varrho_{\mathbb{R}}(R)$ of all $\mathbb{R}$-ideals of $R$ is again an $\mathbb{R}$-ideal of $R$, i.e., $\varrho_{\mathbb{R}}(R)=$ $\bigcup\{I \in \mathcal{I}(R) \cap \mathbb{R}\} \in \mathcal{I}(R) \cap \mathbb{R}$. Moreover, $\varrho_{\mathbb{R}}(R)$ is a subtractive ideal of $R$, and the mapping $\varrho_{\mathbb{R}}: \mathbb{U} \longrightarrow \mathbb{U}$ determined in this way is a radical operator in $\mathbb{U}$.

A subclass $\mathbb{S}$ of a universal class $\mathbb{U}$ is called a semisimple class of $\mathbb{U}[7$, Definition 2.5] iff $\mathbb{S}$ satisfies the following two conditions:

(i) If $R \in \mathbb{S}$, then for every nonzero ideal $I$ of $R$ there exists a nonzero surjective hemiring homomorphism $I \longrightarrow S$ onto $S \in \mathbb{S}$;

(ii) If $R \in \mathbb{U}$ and for every nonzero ideal $I$ of $R$ there exists a nonzero surjective hemiring homomorphism $I \longrightarrow S$ onto $S \in \mathbb{S}$, then $R \in \mathbb{S}$.

As has been shown in 25, Theorems 4.5, 5.4, and 5.5] (see also [7. Theorem 2.6]), each of the three concepts above - radical classes, radical oper- 
ators, and semisimple classes - can serve as a starting point for an internal Kurosh-Amitsur radical theory of semirings/hemirings; and, of course, a hemiring is semisimple iff its radical is zero.

\section{Semimodules and Radical Classes}

We start this section with a developing of an 'external' Kurosh-Amitsur radical theory for semirings/hemirings - a Kurosh-Amitsur radical theory based, at this time, on representations, semimodules, of hemirings - in the spirit of, and by analogy with, the external radical theory for rings (see, for example, [4, Section 3.14]). Hear again, as was mentioned earlier, despite of the natural fact that many corresponding concepts and considerations for radical theories for rings and semirings/hemirings are very similar, in the semiring setting, they are, not surprisingly, significantly more complicated and often - since there is no "pleasure" of the abelian categories, and one must "live in the world without subtraction" - involve original ideas and techniques.

Now, for each hemiring $R$, let $\Sigma_{R}$ be a "chosen" class of left $R$-semimodule $M$ with $R M \neq 0$, and $\Sigma$ be the union of all those $\Sigma_{R}$. Let

$$
\operatorname{ker}\left(\Sigma_{R}\right):=\cap\left\{(0: M)_{R} \mid M \in \Sigma_{R}\right\}
$$

Then, let us single out the following four conditions on the class of semimodules $\Sigma$ which it might satisfy:

(SM1) If $M \in \Sigma_{R / I}$, then $M \in \Sigma_{R}$;

(SM2) If $M \in \Sigma_{R}$ and $I$ is an ideal of $R$ such that $I \subseteq(0: M)_{R}$, then $M \in \Sigma_{R / I}$

(SM3) If $\operatorname{ker}\left(\Sigma_{R}\right)=0$, then $\Sigma_{I} \neq \emptyset$ for all nonzero ideals $I$ of $R$;

(SM4) If $\Sigma_{I} \neq \emptyset$ for each nonzero ideal $I$ of $R$, then $\operatorname{ker}\left(\Sigma_{R}\right)=0$.

Denoting by $\mathcal{F}(\Sigma)$ the class of hemirings $R$ for which the semimodule class $\Sigma_{R}$ contains a faithful semimodule, using Proposition 2.1 and repeating verbatim the proof of [4, Proposition 3.14.2], one obtains its hemiring analog:

Proposition 3.1. (cf. [4, Proposition 3.14.2]) $\mathcal{F}(\Sigma)$ is a regular class.

The following three observations are hemiring versions of the corresponding ring results.

Theorem 3.2. ( $c f$. [4, Theorem 3.14.3]) Let $\Sigma$ be a class of semimodules satisfying conditions (SM1)-(SM3), and $\Re(\Sigma):=\left\{R \in \mathbb{H} \mid \Sigma_{R}=\emptyset\right\}$. Then:

(i) $\Re(\Sigma)$ is a radical class, and $\Re(\Sigma)=\mathcal{U} \mathcal{F}(\Sigma)$;

(ii) $\varrho_{\Re(\Sigma)}(R) \subseteq \operatorname{ker}\left(\Sigma_{R}\right)$ for every hemiring $R$. 
Proof. (i) This can be justified by applying Proposition 2.1 and Theorem 2.3 and repeating verbatim the proof of [4, Theorem 3.14.3 (i)].

(ii) For every hemiring $R$ and semimodule $M \in \Sigma_{R}$, the annihilator $(0: M)$ is obviously a subtractive ideal of $R$, and therefore, by [25, Theorem $2.2]$, there is the semiisomorphism $f: \varrho_{\Re(\Sigma)}(R) /\left(\varrho_{\Re(\Sigma)}(R) \cap(0: M)\right) \longrightarrow$ $\left(\varrho_{\Re(\Sigma)}(R)+(0: M)\right) /(0: M)$ given by $\bar{r} \longmapsto \bar{r}$.

It is clear that $\left(\varrho_{\Re(\Sigma)}(R)+(0: M)\right) /(0: M)$ is an ideal of $R /(0: M) \in$ $\mathcal{F}(\Sigma)$. Assuming that $\left(\varrho_{\Re(\Sigma)}(R)+(0: M)\right) /(0: M) \neq 0$ and using Proposition 2.1 and Theorem 2.3, one has nonzero surjective homomorphisms $g:\left(\varrho_{\Re(\Sigma)}(R)+(0: M)\right) /(0: M) \longrightarrow S \in \mathcal{F}(\Sigma)$ and $g f \pi: \varrho_{\Re(\Sigma)}(R) \longrightarrow S$ $\in \mathcal{F}(\Sigma)$, where $\pi: \varrho_{\Re(\Sigma)}(R) \longrightarrow \varrho_{\Re(\Sigma)}(R) /\left(\varrho_{\Re(\Sigma)}(R) \cap(0: M)\right)$ is the natural surjection. The latter implies that, in contradiction with $(\mathrm{i}), \varrho_{\Re(\Sigma)}(R) \notin$ $\mathcal{U F}(\Sigma)$. Hence, $\left(\varrho_{\Re(\Sigma)}(R)+(0: M)\right) /(0: M)=0$ and $\varrho_{\Re(\Sigma)}(R)+(0: M)=$ $(0: M)$; and, therefore, $\varrho_{\Re(\Sigma)}(R) \subseteq(0: M)$ and $\varrho_{\Re(\Sigma)}(R) \subseteq \operatorname{ker}\left(\Sigma_{R}\right)$.

Theorem 3.3. (cf. [4, Theorem 3.14.4]) Let $\Sigma$ be a class of semimodules satisfying conditions (SM1)-(SM4). Then:

(i) $R$ is $\Re(\Sigma)$-semisimple iff $R$ is semiisomorphic to a subdirect product of hemirings in $\mathcal{F}(\Sigma)$;

(ii) $\varrho_{\Re(\Sigma)}(R)=\operatorname{ker}\left(\Sigma_{R}\right)$ for every hemiring $R$.

Proof. As was shown in Theorem $3.2, \Re(\Sigma)$ is a radical class, and, hence, by [7, Theorem 2.6(c)], the class $\mathbb{S}:=\left\{R \in \mathbb{H} \mid \varrho_{\Re(\Sigma)}(R)=(0)\right\}$ is a semisimple class of $\mathbb{H}$.

(i). $\Longrightarrow$. Let $R$ be $\Re(\Sigma)$-semisimple. Then, $\varrho_{\Re(\Sigma)}(R)=0$, and $R$ has no nonzero ideals in $\Re(\Sigma)$, and hence, $\Sigma_{I} \neq \emptyset$ for all nonzero ideals $I$ of $R$. From the latter and condition (SM4), we have $\operatorname{ker}\left(\Sigma_{R}\right)=\cap_{M \in \Sigma_{R}}(0: M)=0$. By Proposition 2.1, $R /(0: M) \in \mathcal{F}(\Sigma)$ for all $M \in \Sigma_{R}$; and, by [8, Theorem 6.3 (b)], there exists the semiisomorphism $f: R \longrightarrow \prod_{M \in \Sigma_{R}} R /(0: M)$ given by $r \longmapsto\left([r]_{\equiv_{(0: M)}}\right)_{M \in \Sigma_{R}}$.

$\Longleftarrow$. Let $R$ be semiisomorphic to a subdirect product $T=\prod_{i \in I}^{s u b} R_{i}$ of hemirings $R_{i} \in \mathcal{F}(\Sigma)$. Then, $\operatorname{ker}\left(\Sigma_{R_{i}}\right)=0$, and hence, by Theorem 3.2 (ii), $R_{i}$ is $\Re(\Sigma)$-semisimple, and $R_{i} \in \mathbb{S}$ for all $i \in I$. By [8, Theorem 4.3 (a)] $\mathbb{S}$ is subdirectly closed in $\mathbb{H}$ and, therefore, $T=\prod_{i \in I}^{s u b} R_{i} \in \mathbb{S}$, too. By [8, Theorem 3.7(b)], $\mathbb{S}$ is also inverse semiisomorphically closed in $\mathbb{H}$ (i.e., if there exists a semiisomorphism $\varphi: R \rightarrow T \in \mathbb{S}$, then $R \in \mathbb{S}$ ) and, hence, $R \in \mathbb{S}$, i.e., $R$ is $\Re(\Sigma)$-semisimple.

(ii). Using Theorem 3.2 (ii), we only need to show that $\varrho_{\Re(\Sigma)}(R) \supseteq$ $\operatorname{ker}\left(\Sigma_{R}\right)$. Indeed, since $R / \varrho_{\Re(\Sigma)}(R)$ is clearly $\Re(\Sigma)$-semisimple, as in the proof of the first part of (i), we get the semiisomorphism $f: R / \varrho_{\Re(\Sigma)}(R) \longrightarrow$ $\prod_{M \in \Sigma_{R / \varrho_{\Re(\Sigma)}(R)}^{s u b}}^{s / \varrho_{\Re(\Sigma)}}(R) /(0: M)_{R / \varrho_{\Re(\Sigma)}(R)}$ with $R / \varrho_{\Re(\Sigma)}(R) /(0: M)_{R / \varrho_{\Re(\Sigma)}(R)} \in \mathcal{F}(\Sigma)$; and, hence, $R / \varrho_{\Re(\Sigma)}(R)$ is semiiso- 
morphic to a subdirect product $f\left(R / \varrho_{\Re(\Sigma)}(R)\right)$ of hemirings $R / \varrho_{\Re(\Sigma)}(R) /(0$ : $M)_{R / \varrho_{\Re(\Sigma)}(R)}$. Applying Proposition 2.1(iv) and [8, Theorem 2.3], one gets $(0: M)_{R / \varrho_{\Re(\Sigma)}(R)} \cong(0: M)_{R} / \varrho_{\Re(\Sigma)}(R)$ and $R / \varrho_{\Re(\Sigma)}(R) /(0: M)_{R / \varrho_{\Re(\Sigma)}(R)} \cong$ $R /(0: M)_{R}$. Hence, $R /(0: M)_{R} \in \mathcal{F}(\Sigma)$, and therefore there is a faithful left $R /(0: M)_{R^{-}}$semimodule $N$ in $\Sigma_{R /(0: M)_{R}}$; and for condition (SM1), $N \in$ $\Sigma_{R}$. Now, applying again Proposition 2.1, we get $(0: N)_{R} /(0: M)_{R}=$ $(0: N)_{R /(0: M)_{R}}=0$, and hence, $\operatorname{ker}\left(\Sigma_{R}\right) \subseteq(0: N)_{R}=(0: M)_{R}$ and $\operatorname{ker}\left(\Sigma_{R}\right) \subseteq \cap(0: M)_{R} \subseteq \varrho_{\Re(\Sigma)}(R)$.

The next observation, actually inverse to Theorems 3.2 and 3.3, shows that every radical class can be obtained from a suitable class of semimodules $\Sigma$ satisfying conditions (SM1)-(SM4).

Theorem 3.4. (cf. [4, Theorem 3.14.5]) Let $\mathbb{R} \subseteq \mathbb{H}$ be a radical class, $\Sigma_{R}=\left\{{ }_{R} M \in\left|{ }_{R} \mathcal{M}\right| \mid R M \neq 0 \& \varrho_{\mathbb{R}}(R /(0: M))=(0)\right\}$ for every $R \in \mathbb{H}$, and $\Sigma=\cup \Sigma_{R}$. Then:

(i) $\Sigma$ satisfies conditions (SM1)-(SM4);

(ii) $\mathbb{R}=\Re(\Sigma)$.

Proof. (i) Using Proposition 2.1 and repeating verbatim the proofs for the corresponding ring conditions (M1) and (M2) in [4, Theorem 3.14.5], we get that the class $\Sigma$ satisfies (SM1) and (SM2).

To establish (SM3) for the class $\Sigma$, suppose that $\operatorname{ker}\left(\Sigma_{R}\right)=0$ and $I$ is a nonzero ideal of $R$, and therefore, $I M \neq 0$ for some semimodule $M \in \Sigma_{R}$. By [7, Theorem 2.6(c)], the class of semimodules $\mathbb{S}=\left\{R \in \mathbb{H} \mid \varrho_{\mathbb{R}}(R)=\right.$ $(0)\}$ is a semisimple class of $\mathbb{H}$. Then, by [25, Theorem 2.2] there exists a semiisomorphism $f: I /(0: M)_{I}=I /\left((0: M)_{R} \cap I\right) \longrightarrow\left(I+(0: M)_{R}\right) /(0:$ $M)_{R}$, where $\left(I+(0: M)_{R}\right) /(0: M)_{R}$ is an ideal of $R /(0: M)_{R} \in \mathbb{S}$. Whence, by [8, Theorem 3.7(a)] $\left(I+(0: M)_{R}\right) /(0: M)_{R} \in \mathbb{S}$, and since by [8, Theorem 3.7(b)] $\mathbb{S}$ is inverse semiisomorphically closed in $\mathbb{H}$, we have $I /(0: M)_{I} \in \mathbb{S}$ and, hence, $\varrho_{\mathbb{R}}\left(I /(0: M)_{I}\right)=(0)$. From the latter, one has that $M \in \Sigma_{I}$ and, therefore, $\Sigma$ satisfies (SM3), too.

The fact that $\Sigma$ satisfies (SM4) can be establish by verbatim repeating the proof of the condition (M4) for $\Sigma$ in [4, Theorem 3.14.5] and using Theorem 2.2.

(ii) Suppose $R \in \mathbb{R}$ and $M$ is a left $R$-semimodule having $\varrho_{\mathbb{R}}(R /(0$ : $M))=(0)$. Then, by Theorem $2.2 R /(0: M) \in \mathbb{R}$ and, hence, $R /(0: M)=$ $\varrho_{\mathbb{R}}(R /(0: M))=(0)$. Therefore, as $(0: M)$ is a subtractive ideal of $R$, we have $R=(0: M)$ and, hence, $R M=0$. From the latter we conclude that $\Sigma_{R}=\emptyset$, and, therefore, $R \in \Re(\Sigma)$, i.e., $\mathbb{R} \subseteq \Re(\Sigma)$.

Now suppose $R \notin \mathbb{R}$. From Theorem 2.2 it follows that there exists a nonzero surjective homomorphism $f: R \longrightarrow S$ with $\varrho_{\mathbb{R}}(S)=(0)$, and, therefore, $S$ is semiisomorphic $R / I$, where $I=\operatorname{Ker}(f)$, and applying [8, 
Theorem 3.7(b)], one gets $\varrho_{\mathbb{R}}(R / I)=(0)$. Then, consider the Dorroh extension $(R / I)^{1}$ of $R / I$ : It is obvious that $(R / I)^{1}$ is a left $R / I$-semimodule with $\left(0:(R / I)^{1}\right)_{R / I}=(0)$; hence, $(R / I) /\left(0:(R / I)^{1}\right)_{R / I}=R / I$ is $\mathbb{R}$-semisimple. The latter implies that $(R / I)^{1} \in \Sigma_{R / I}$; moreover, since $\Sigma$ satisfies (SM1), $(R / I)^{1} \in \Sigma_{R}$, and, hence, $R \notin \Re(\Sigma)$. Thus, $\Re(\Sigma) \subseteq \mathbb{R}$.

As Theorem 3.4 shows, any radical class might be, in principle, "externally" described by means of the class of suitable presentations/semimodules of hemirings. However, in this theorem the class of the corresponding

semimodules $\Sigma$, constructed straightforwardly from the radical itself, is the greatest of all possible classes describing a given radical class. Therefore, it is more important and interesting to be able to find a smaller than $\Sigma$ class of semimodules describing the same radical class and/or having a nice practical characterization. In some important cases this can be successfully done and to illustrate that, in the following two subsections of this section, we consider two analogs of the classical ring radicals - the Jacobson and the Brown-McCoy radicals-for semirings.

\subsection{The Jacobson Type Radicals of Semirings}

A nonzero cancellative left semimodule $M$ over a hemiring $R$ is irreducible [9, Definition 5] if, for an arbitrarily fixed pair of elements $m_{1}, m_{2}$ of $M$ with $m_{1} \neq m_{2}$ and any $m \in M$, there exist $r_{1}, r_{2} \in R$ such that

$$
m+r_{1} m_{1}+r_{2} m_{2}=r_{1} m_{2}+r_{2} m_{1} \text {. }
$$

It is easy to see that $R M \neq 0$ for any irreducible left $R$-semimodule $M$. And a nonzero cancellative left semimodule $M$ over a hemiring $R$ is semi-irreducible [9, p. 412] if $R M \neq 0$ and there are no subtractive subsemimodules of $M$ except $M$ itself and the zero one. Finally, we call a left $R$-semimodule $M$ simple if $R M \neq 0$ and there are only trivial subsemimodules of, as well as congruences on, $M$.

Proposition 3.5. (cf. [4, Theorem 3.14.6]) For every hemiring $R \in \mathbb{H}$, let $\Sigma_{R}$ denote the corresponding class of semimodules in each of the cases:

(i) $\Sigma_{R}:=\left\{\left.{ }_{R} M \in\right|_{R} \mathcal{M}|| M\right.$ is irreducible $\}$,

(ii) $\Sigma_{R}:=\left\{{ }_{R} M \in\left|{ }_{R} \mathcal{M}\right| \mid M\right.$ is semi-irreducible $\}$,

(iii) $\Sigma_{R}:=\left\{\left.{ }_{R} M \in\right|_{R} \mathcal{M}|| M\right.$ is simple $\}$.

Then, for each of these cases, $\Sigma:=\cup \Sigma_{R}$ satisfies conditions (SM1)-(SM4).

Proof. First, for each of the cases (i)-(iii), one may easily verify that $\Sigma$ satisfies (SM1) and (SM2).

(i). Suppose $\operatorname{ker}\left(\Sigma_{R}\right)=0$ for a hemiring $R$, and $I$ is a nonzero ideal of $R$. Then, $I M \neq 0$ for some irreducible left $R$-semimodule $M$ and by 
[9, Lemma 4(1)] $M$ is an irreducible left $I$-semimodule; so, $\Sigma_{I} \neq \emptyset$ and $\Sigma$ satisfies (SM3).

Now suppose that $\Sigma_{I} \neq \emptyset$ for all nonzero ideals $I$ of a hemiring $R$, and $B:=\operatorname{ker}\left(\Sigma_{R}\right) \neq 0$. Then, $B M=0$ for all irreducible left $R$-semimodule $M$. Since $\Sigma_{B} \neq \emptyset$, there exists an irreducible left $B$-semimodule $N$, and hence, $B N \neq 0$. Whence by [9, Lemma 4(2)] $B N$ is an irreducible left $R$-semimodule, and therefore, $B(B N)=0$. However, by [9, Lemma 3] $B N$ is also an irreducible left $B$-semimodule, and hence, $B(B N) \neq 0$. Thus, $\operatorname{ker}\left(\Sigma_{R}\right)=0$, and $\Sigma$ satisfies (SM4), too.

(ii). Using [9, Lemma 4(1)], the conditions (SM3) for $\Sigma$ can be established in the same fashion as in (i).

To verify (SM4) for the class $\Sigma$, assume that $\Sigma_{I} \neq \emptyset$ for all nonzero ideals $I$ of a hemiring $R$ and $B:=\operatorname{ker}\left(\Sigma_{R}\right) \neq 0$. So, $B N \neq 0$ for some a semiirreducible left $B$-semimodule $N \in \Sigma_{B}$. As was shown in [9, p. 416], on the semi-irreducible semimodule $N$ there exists a maximal semimodule congruence $\rho$ such that the $B$-semimodule $N^{\prime}:=N / \rho$ is an reducible semimodule. Whence, $B N^{\prime} \neq 0$ and, applying [9, Lemma 4(2) and Corollary on p. 413], $B N^{\prime}$ is a semi-irreducible left $R$-semimodule; and therefore, $B\left(B N^{\prime}\right)=0$. However, by [9, Lemma 4(1)] $B N^{\prime}$ is also an irreducible left $B$-semimodule, and hence, $B\left(B N^{\prime}\right) \neq 0$. Thus, $\operatorname{ker}\left(\Sigma_{R}\right)=0$, and $\Sigma$ satisfies (SM4), too.

(iii). Suppose $\operatorname{ker}\left(\Sigma_{R}\right)=0$ for a hemiring $R$, and $I$ is a nonzero ideal of $R$. Then, $I M \neq 0$ for some simple left $R$-semimodule $M$; and for a nonzero element $m \in M$, the semimodule ${ }_{R} M$ contains two subsemimodules-Im and $K:=\{x \in M \mid I x=0\}$; and since ${ }_{R} M$ is simple, $K=0$ or $K=M$. However, the latter is not a case as $I M \neq 0$, and hence, $K=0$ and $I m=M$, and therefore, the left semimodule ${ }_{I} M$ has only trivial $I$-subsemimodules.

Next, let $\rho$ be a congruence on ${ }_{I} M$; and consider the congruence $\theta$ on ${ }_{R} M$, where $m \theta m^{\prime}$ iff $\left(a m, a m^{\prime}\right) \in \rho$ for all $m, m^{\prime} \in M$ and $a \in I$. It is clear that $\theta$ is indeed a congruence on ${ }_{R} M$ and $\rho \subseteq \theta$; and since ${ }_{R} M$ is simple, $\theta=\Delta_{R} M$ or $\theta=M^{2}$. If $\theta=\Delta_{R} M$, then, obviously, $\rho=\Delta_{I} M$, too. If $\theta=M^{2}$, then $(m, 0) \in \theta$ and $(a m, 0) \in \rho$ for all $a \in I$ and for every nonzero $m \in M$; however, as was shown above, $\operatorname{Im}=M$, and hence, $(x, 0) \in \rho$ for all $x \in M$. Therefore, ${ }_{I} M$ is congruence -simple, i.e., there are only the trivial congruences on ${ }_{I} M$, and $\Sigma_{I} \neq \emptyset$, and the class $\Sigma$ satisfies (SM3).

To verify (SM4) for the class $\Sigma$, consider a hemiring $R$ having $\Sigma_{I} \neq \emptyset$ for all nonzero ideals $I$ of $R$ and with $B:=\operatorname{ker}\left(\Sigma_{R}\right) \neq 0$. So, $B M=0$ for all simple left $R$-semimodules, and there exist a simple left $B$-semimodule $X$ and an element $x \in X$ such that $B x=X \neq 0$, and hence, $X=B X$. We may extend ${ }_{B} X$ to a left $R$-semimodule ${ }_{R} X$ defining

$$
r \Sigma b_{i} x_{i}=\Sigma\left(r b_{i}\right) x_{i}
$$


for all $b_{i} \in B, x_{i} \in X$ and $r \in R$. Indeed, if $\Sigma b_{i} x_{i}=\Sigma b_{j}^{\prime} x_{j}^{\prime}\left(b_{i}, b_{j}^{\prime} \in B\right.$, $\left.x_{i}, x_{j}^{\prime} \in X\right)$, then, since $X$ is a left $B$-semimodule,

$$
b\left(r \sum b_{i} x_{i}\right)=b \Sigma\left(r b_{i}\right) x_{i}=\Sigma\left(b r b_{i}\right) x_{i}
$$

for every $b \in B$; moreover,

$$
\Sigma\left(b r b_{i}\right) x_{i}=(b r) \Sigma b_{i} x_{i}=b r \Sigma b_{j}^{\prime} x_{j}^{\prime}=\Sigma b r b_{j}^{\prime} x_{j}^{\prime}=b \Sigma r b_{j}^{\prime} x_{j}^{\prime}=b\left(r \sum b_{j}^{\prime} x_{j}^{\prime}\right),
$$

and so, $b\left(r \Sigma b_{i} x_{i}\right)=b\left(r \Sigma b_{j}^{\prime} x_{j}^{\prime}\right)$. Consider the congruence $\alpha$ on ${ }_{B} X$, where, for any $u, v \in X$, the ordered pair $(u, v) \in \alpha$ iff $b u=b v$ for all $b \in B$. Since ${ }_{B} X$ is congruence-simple, $\alpha=\Delta_{B} X$ or $\alpha=X^{2}$. The latter is not a case since otherwise $(u, 0) \in \alpha$ for every $u \in X$, i.e., $b u=0$ for all $b \in B$, and hence, $B x=X=0$. Hence, $\alpha=\Delta_{B} X$. Whence, $r \sum b_{i} x_{i}=r \Sigma b_{j}^{\prime} x_{j}^{\prime}$. Thus, the 'scalar multiplication' by elements of $R$ is well-defined, and it is a routine to verify that $X$ is a left $R$-semimodule.

Now, since $R X \supseteq B X \neq 0$, we have $R X \neq 0$, and for $X$ is a simple left $B$-semimodule, $X$ is a simple left $R$-semimodule, too; and hence, one gets a contradiction with $B X=0$. Therefore, there must be $\operatorname{ker}\left(\Sigma_{R}\right)=0$, and the class $\Sigma$ satisfies (SM4), too.

Now consider an hemiring analog of the Jacobson radical for rings introduced by B. Bourne in [3]. First, recall [3, Definition 3] that a right ideal $I$ of a hemiring $R$ is right semiregular if, for every pair of elements $i_{1}, i_{2} \in I$, there exist elements $j_{1}$ and $j_{2}$ in $I$ such that $i_{1}+j_{1}+i_{1} j_{1}+i_{2} j_{2}=i_{2}+j_{2}+i_{1} j_{2}+i_{2} j_{1}$. By [3, Theorems 3 and 4], the sum $J(R)$ of all the right semiregular ideals of a hemiring $R$ forms a right semiregular two-sided ideal, which is called the Jacobson radical of the hemiring $R$. Also, by [3, Theorems 5 and 6], the mapping $\varrho: \mathbb{H} \longrightarrow \mathbb{H}$ given by $R \longmapsto J(R)$ is, in fact, a radical operator in $\mathbb{H}$. Therefore, from Theorem 2.4 and [7, Theorem 2.6], it follows that the class

$$
\mathcal{J}:=\{R \in \mathbb{H} \mid J(R)=R\}
$$

is a radical class, the Jacobson-Bourne radical class, of $\mathbb{H}$. Using these observations, 9, Definitions 6 and 6', and Theorem 8], Theorems 3.2 and 3.3, and Proposition 3.5, we obtain the following hemiring analog of 4, Example 3.14.12]:

Example 3.6. Let $\Sigma$ be one of the classes $-\cup_{R \in \mathbb{H}}\left\{{ }_{R} M \in{ }_{R} \mathcal{M}|| M\right.$ is irreducible $\}$ or $\cup_{R \in \mathbb{H}}\left\{\left.{ }_{R} M \in\right|_{R} \mathcal{M}|| M\right.$ is semi-irreducible $\}$ - of semimodules over hemirings. Then, $\Re(\Sigma)=\mathcal{J}$, the Jacobson-Bourne radical class.

There is another very natural hemiring analog of the Jacobson radical for rings, namely: Applying Theorems 3.2 and 3.3 and Proposition 3.9, we obtain that for the class of semimodules $\Sigma=\cup_{R \in \mathbb{H}}\left\{{ }_{R} M \in\left|{ }_{R} \mathcal{M}\right| \mid M\right.$ is 
simple $\}$, the class $\Re(\Sigma)$ is a radical class and $\varrho_{\Re(\Sigma)}(R)=\operatorname{ker}\left(\Sigma_{R}\right) \stackrel{\text { def }}{=} J_{s}(R)$ is the radical for every hemiring $R$. Obviously, on the subclass of all rings of the class $\mathbb{H}$ both radicals, $J(R)$ and $J_{s}(R)$, coincide. However, in general, as the following example demonstrates, they are different.

Example 3.7. Consider the Boolean semiring $\mathbf{B}=\{0,1\}$. It is clear that $\mathbf{B}$ is a simple $\mathbf{B}$-semimodule and $(0: \mathbf{B})_{\mathbf{B}}=0$, and therefore, $J_{s}(\mathbf{B})=$ 0 . However, there are no irreducible $\mathbf{B}$-semimodules: Indeed, if ${ }_{\mathbf{B}} M$ is a irreducible semimodule, then $\mathbf{B} M \neq 0$ and $(M,+)$ is a cancellative monoid; therefore, $1 . m=(1+1) m=1 . m+1 . m$, and hence, $1 . m=0$ for each $m \in M$, i.e., $\mathbf{B} M=0$. Thus, $J(\mathbf{B})=\mathbf{B}$.

As it will be shown later in Proposition 4.8, this example is a particular case of the general observation: for commutative hemirings $R$, there always takes place the inclusion $J_{s}(R) \subseteq J(R)$. In light of these observations, it is natural to state the following, in our view interesting, problem.

Problem 1. Describe the subclass of all hemirings $R$ of the class $\mathbb{H}$ with $J_{s}(R) \subseteq J(R)$, particularly, with $J(R)=J_{s}(R)$.

A hemiring $R$ is called primitive [9, Definition 7] iff it has a faithful irreducible left semimodule. Using Theorem 3.3 and Example 3.6, one gets the following result, removing the assumption of the additive cancellation on primitive hemirings in [19, Theorem 3.3, p. 12] and, hence, resolving the question left open there:

Corollary 3.8. A hemiring $R$ is J-semisimple, i.e., $J(R)=0$, iff $R$ is semiisomorphic to a subdirect product of primitive hemirings.

Next, as an easy corollary of this result and our "external" approach to radicals of hemirings, we immediately deduce Theorem 3.4 and Corollary 3.5 of [19]:

Corollary 3.9. (cf. [19, Theorem 3.4, and Corollary 3.5]) A nonzero additively regular hemiring $R$ is $J$-semisimple iff $R$ is a ring isomorphic to a subdirect product of primitive rings.

Proof. First, it is clear that any additive idempotent of a hemiring $S$ acts on any irreducible $S$-semimodule like $0 \in S$. Whence, the zero is the only additive idempotent of a primitive additively regular hemiring; and, therefore, any primitive additively regular hemiring is, in fact, a ring. From this observation, Corollary 3.8, and the obvious observation that a subdirect product of hemirings which are rings is a ring, we end the proof.

A hemiring $R$ is congruence-simple [1] (also, [2], [24]) if the diagonal, $\triangle_{R}$, and universal, $R^{2}$, congruences are the only congruences on $R$. In the 
next theorem, we, in particular, give a complete description of $J$-semisimple, congruence-simple hemirings.

Theorem 3.10. (i) Let $R$ be a congruence-simple hemiring. Then $R$ is a $J$-radical (i.e., $J(R)=R$ ) or primitive hemiring.

(ii) A J-semisimple (i.e., $J(R)=0$ ) hemiring $R$ is congruence-simple iff $R$ is a simple ring.

Proof. (i). As is well-known (see, for example, Theorem 2.4), $J(R)$ is a subtractive ideal of $R$. But, by [15, Proposition 4.4], $R$ has only two trivial subtractive ideals, and therefore, $J(R)=R$ or $J(R)=0$. If $J(R)=0$, then by Corollary 3.8, there exists a hemiring semiisomorphism $f: R \longrightarrow$ $\prod_{i \in I}^{s u b} R_{i}$, where $R_{i}, i \in I$, are primitive hemirings, and hence, $R_{i} \neq 0, i \in I$. Then, there is a nonzero surjective homomorphism $g:=\left.\pi_{i}\right|_{T} f: R \longrightarrow$ $\prod_{i \in I}^{s u b} R_{i} \longrightarrow R_{i}$ which induces the natural congruence ' $\equiv$ ' on $R$ defined for all $r, s \in R$ by: $r \equiv s \Longleftrightarrow g(r)=g(s)$. Because $R$ is congruence-simple, we immediately get that $g$ is injective as well, and therefore, $R$ is isomorphic to a primitive hemiring $R_{i}$.

(ii). $\Longrightarrow$. By (i), $R$ is a primitive hemiring, and hence, there exists a faithful irreducible left $R$-semimodule $M$. By [33, Proposition 3.1], congruence-simpleness of a hemiring $R$ implies that either $R$ is a simple ring or the reduct $(R,+, 0)$ is an idempotent monoid. In the latter case, because $(M,+)$ is a cancellative monoid, $R M=0$, and hence, the semimodule $M$ is not irreducible. Thus, $R$ is a simple ring.

$\Longleftarrow$. It is obvious.

Let $M$ be a join semilattice with zero; and following [33], for any elements $a, b \in M$, consider the following mappings on $M$ :

$$
e_{a, b}(x):=\left\{\begin{array}{l}
0 \text { if } x \leq a, \\
b \text { otherwise }
\end{array}(x \in M)\right. \text {. }
$$

As was shown in [33, Lemma 2.2], $e_{a, b} \in \operatorname{End}(M)$ for all $a, b \in M$. Then, let $\mathbf{F}_{M}$ be the submonoid of $(\operatorname{End}(M),+, 0)$ generated by the endomorphisms $e_{a, b}, a, b \in M$. It is easy to see (see, also, [33, Lemma 2.2] and [16, Lemma 3.1]) that $\mathbf{F}_{M}$ is a left ideal of $\operatorname{End}(M)$, which is an ideal when $M$ is finite. A subhemiring $R$ of $\operatorname{End}(M)$ is said to be dense [33] (also, [10, p. 154]) if it contains $\mathbf{F}_{M}$. We conclude this subsection with a characterization of finite additively-idempotent $J_{s}$-semisimple hemirings.

Theorem 3.11. A finite additively idempotent hemiring $R$ is $J_{s}$-semisimple (i.e., $J_{s}(R)=0$ ) iff it is semiisomorphic to a subdirect product of some hemirings $S_{i}(i \in I)$ such that each of the hemirings $S_{i}(i \in I)$, in turn, is isomorphic to a dense subhemiring of the endomorphism hemiring $\operatorname{End}\left(M_{i}\right)$ $(i \in I)$ of a finite semilattice $M_{i}(i \in I)$ with zero. 
Proof. $\Longleftarrow$. By [10, Corollary 5.4], for each $i \in I$, there is a simple left $S_{i}$-semimodule $M_{i}$ such that the map $\varphi: S_{i} \longrightarrow \operatorname{End}\left(M_{i},+\right)$, defined for all $s \in S_{i}$ and $m \in M_{i}$ by $\varphi(s)(m)=s m$, is an injective homomorphism of hemirings, and hence, $\left(0: M_{i}\right)_{S_{i}}=0$. Whence by Theorem 3.3, $J_{s}(R)=0$.

$\Longrightarrow$. By Theorem 3.3, the hemiring $R$ is semiisomorphic to a subdirect product of finite additively-idempotent hemirings $R_{i}(i \in I)$ such that, for each $i \in I$, there exists a simple faithful left $R_{i}$-semimodule $M_{i}$ for which, of course, $\left(M_{i},+\right)$ is a finite idempotent monoid and $M_{i}=R_{i} m$ for any nonzero $m \in M_{i}$. Thus, there exist the hemiring homomorphism $\varphi_{i}: R_{i} \longrightarrow$ $\operatorname{End}\left(M_{i},+\right)$ defined for all $r \in R_{i}$ and $m \in M_{i}$ by $\varphi(r)(m)=r m$ and having $\operatorname{ker}\left(\varphi_{i}\right):=\varphi_{i}^{-1}(0)=\left(0: M_{i}\right)_{R_{i}}=0$, as well as the corresponding congruence " $\equiv_{\varphi_{i}}$ " on $R_{i}$ such that $r \equiv \varphi_{i} r^{\prime} \Longleftrightarrow \varphi_{i}(r)=\varphi_{i}\left(r^{\prime}\right)$ for all $r, r^{\prime} \in R_{i}$ and, hence, the injective homomorphism $\psi: R_{i} / \equiv{ }_{\varphi_{i}} \longrightarrow \operatorname{End}\left(M_{i},+\right)$ such that $\psi(\bar{r})=\varphi_{i}(r)$ for all $r \in R_{i}$. For the natural projection $\pi: R_{i} \longrightarrow R_{i} / \equiv{ }_{\varphi_{i}}$, obviously, $\operatorname{ker}(\pi):=\pi^{-1}(\overline{0})=\left(0: M_{i}\right)_{R_{i}}=0$, and therefore, $\pi$ is a semiisomorphism and $R$ is semiisomorphic to a subdirect product of the hemirings $S_{i}:=R_{i} / \equiv{ }_{\varphi_{i}}$. Also, it is clear that $M_{i}$ with the 'scalar multiplication' given by $\bar{r} m=r m$ for all $m \in M$ and $r \in R_{i}$ is a finite simple left $S_{i}$-semimodule. Now, for the homomorphism $\psi: R_{i} / \equiv_{\varphi_{i}} \longrightarrow \operatorname{End}\left(M_{i},+\right)$ is injective and [10, Theorem 5.3], we conclude that $S_{i}$ is isomorphic to a dense subhemiring of the endomorphism hemiring $\operatorname{End}\left(M_{i}\right)$ of a finite semilattice $M_{i}$ with zero.

\subsection{The Brown-McCoy Radical of Semirings}

The Brown-McCoy radical for hemirings constitutes another important hemiring analog of a classical radical for rings. Consider the subclass of ideal-simple semirings $\mathbb{S}:=\{R \in \mathbb{H} \mid R$ is an ideal-simple semiring $\}$ of the class $\mathbb{H}$. It is obvious that subclass $\mathbb{S}$ is a regular class of $\mathbb{H}$, and therefore, by Theorem 2.3, the class $\mathcal{U} \mathbb{S}=\{R \in \mathbb{H} \mid R$ has no nonzero homomorphic images in $\mathbb{S}\}$ forms a radical class, the Brown-McCoy radical class, of $\mathbb{H}$; and by $\mathcal{R}_{B M}(R)$ we will denote the corresponding Brown-McCoy radical of a hemiring $R$. Because for any semiring $R$, obviously, there exists a maximal congruence $\rho$ on $R$ such that $R / \rho$ is a congruence-simple semiring, it is clear that a hemiring $R$ is a Brown-McCoy radical hemiring, i.e. $\mathcal{R}_{B M}(R)=R$, iff there is no nonzero homomorphic images of $R$ in the class of all simple semirings.

The Brown-McCoy radicals for rings and some other algebraic systems can be characterized in terms of some special congruences (see, for instance, [4, Section 4.8] and [21, p. 430] and references there). In our next result, we present such a characterization for hemirings belonging to a fairly large 
class - including, in particular, all commutative hemirings and rings - of hemirings. For that we need natural hemiring analogs of some well-known for rings notions.

Given a hemiring $R$ and element $r \in R$, consider two congruences, $\rho_{G(r)}$ and $\rho_{F_{1}(r)}$, on $R$, generated by two subsets

$$
\begin{aligned}
G(r): & =\left\{\left(r x+\sum_{i=1}^{n} y_{i} r z_{i}, x+\sum_{i=1}^{n} y_{i} z_{i}\right) \mid n \in \mathbb{N}, x, y_{i}, z_{i} \in R\right\} \text { and } \\
F_{1}(r): & =\{(r x+y r, x+y) \mid x, y \in R\}
\end{aligned}
$$

of $R^{2}$, respectively. Further, following [4, Section 4.8], we say that the element $r$ is G-regular $\left(F_{1}\right.$-regular $)$ if $(r, 0) \in \rho_{G(r)}\left((r, 0) \in \rho_{F_{1}(r)}\right)$; and a hemiring $R$ is called $G$-regular ( $F_{1}$-regular) if every element $r \in R$ is $G$ regular $\left(F_{1}\right.$-regular $)$. Now we are ready to extend the characterization of the Brown-McCoy radical for rings [4, Theorem 4.8.2] to the subclasses of subtractive, commutative, and lattice-ordered, hemirings.

Theorem 3.12. Let $R$ be a subtractive (or commutative, or lattice-ordered) hemiring. Then, the following statements are equivalent:

(i) $R$ is a Brown-McCoy radical hemiring, i.e., $R \in \mathcal{U} \mathbb{S}$;

(ii) $R$ is G-regular;

(iii) $R$ is $F_{1}$-regular.

Proof. (i) $\Longrightarrow$ (ii). Let $(r, 0) \notin \rho_{G(r)}$ for some element $r \in R$. By the Zorn's Lemma, there exists a maximal congruence $\rho$ on $R$ such that $\rho_{G(r)} \subseteq \rho$ and $(r, 0) \notin \rho$. In fact, $\rho$ is a maximal congruence on $R$ : Indeed, since $(r, 0) \in \theta$ for any properly containing $\rho$ congruence $\theta$ on $R$ and $(r x, 0),(r x, x) \in \theta$ for all $x \in R$, one has $(x, 0) \in \theta$ for all $x \in R$, and hence, $R^{2} \subseteq \theta$. So, $S=R / \rho$ is a congruence-simple hemiring with $(r, 0) \notin \rho$, i.e., $\bar{r} \neq \overline{0}$. For $(r x, x) \in$ $G(r) \subseteq \rho$, we have $\bar{r} \bar{x}=\bar{x}$ for all $x \in R$, and hence, $\bar{r}$ is a left identity on $S$. We claim that, actually, $\bar{r}$ is an identity on $S$. Indeed, for the relation $\gamma:=\left\{(x, y) \in S^{2} \mid x \bar{r}=y \bar{r}\right\}$ on $S$, it is easy to see that $(x+a, y+a) \in \gamma$ and $(a x, a y) \in \gamma$ for any $(x, y) \in \gamma$ and $a \in S$; moreover, since $x \bar{r}=y \bar{r}$ and $\bar{r} a=a$, we have $x a=x(\bar{r} a)=(x \bar{r}) a=(y \bar{r}) a=y(\bar{r} a)=y a$, and therefore, ( $x a, y a) \in \gamma$ and, hence, $\gamma$ is a congruence on $S$. Thus, $\gamma=\triangle_{S}$ or $\gamma=S^{2}$. In the latter, however, $(\bar{r}, \overline{0}) \in \gamma$ and, hence, $\bar{r}=\bar{r} \bar{r}=\overline{0} \bar{r}=\overline{0}$ what contradicts with $\bar{r} \neq \overline{0}$. Whence, $\gamma=\triangle_{S}$. As obviously $(y \bar{r}, y) \in \gamma=\triangle_{S}$ for all $y \in S$, one gets that $y \bar{r}=y$ for all $y \in S$ and $\bar{r}$ is also a right identity on $S$. Now consider the following three cases.

If $R$ is a commutative hemiring, then $S$ is a commutative congruencesimple semiring, and therefore, by [23, Theorem 3.2] (or [1, Theorem 10.1]), $S$ is either a field or isomorphic to the Boolean semifield $\mathbf{B}:=\{0,1\}$. Anyway, $S$ is an ideal-simple semiring. 
If the hemiring $R$ is subtractive hemiring, then $S$ is also subtractive, and hence, a subtractive congruence-simple semiring, which, by [15, Proposition 4.4], is ideal-simple, too.

Finally, let $R$ be a lattice-ordered hemiring. Then, $(R,+, 0)$ and $(S,+, \overline{0})$ are additively idempotent monoids; and let $I \subseteq S$ be a nonzero ideal of $S$. For $S$ is congruence-simple, the Bourne congruence " $\equiv_{I}$ " on $S$ coincides with $S^{2}$. So, $\bar{r} \equiv_{I} \overline{0}$, i.e., there exist elements $a, b \in R$ such that $\bar{a}, \bar{b} \in I$ and $\bar{b}=\bar{r}+\bar{a}$. For $\bar{a}=\overline{r a}=\overline{r a} \leq \overline{r \wedge a} \leq \bar{r}$, it follows that $\bar{b}=\bar{r}+$ $\bar{a}=\bar{r} \in I$, and hence, $I=S$; and therefore, $S$ is an ideal-simple semiring in this case, too.

Thus in all three cases, we have shown that $R$ has a nonzero homomorphic image $S$ which is a simple semiring, and therefore, $R \notin \mathcal{U} \mathbb{S}$.

(ii) $\Longrightarrow$ (iii). It is obvious since $\rho_{G(r)} \subseteq \rho_{F_{1}(r)}$ for all $r \in R$.

(iii) $\Longrightarrow$ (i). In fact, this implication is true for any hemiring $R$, because if $R$ is a $F_{1}$-regular hemiring and $\tau$ is a congruence on $R$ such that $\tau \neq R^{2}$, then the factor hemiring $S=R / \tau$ has no identity element: Indeed, if for some $e \in R$ the element $\bar{e} \in S$ is an identity of $S$, then, as clearly $F_{1}(e) \subseteq \tau$, one has that $(e, 0) \in \rho_{F_{1}(e)} \subseteq \tau$. Thus, $R$ has no nonzero homomorphic images in $\mathbb{S}$, and therefore, $\mathcal{R}_{B M}(R)=R$.

In contrast to rings, the implication (i) $\Longrightarrow$ (iii) in Theorem 3.12 in general is not true for hemirings, namely:

Example 3.13. A semiring End $(M)$ of endomorphisms of a non-distributive finite lattice $M$ is not $F_{1}$-regular semiring with $\mathcal{R}_{B M}(\operatorname{End}(M))=\operatorname{End}(M)$.

Proof. By [33, Proposition 2.3], End $(M)$ is a congruence-simple semiring, and therefore, any nonzero surjective homomorphism $\alpha: \operatorname{End}(M) \longrightarrow S$ to an ideal-simple semiring $S$, would be an isomorphism, and hence, $\operatorname{End}(M)$ would be a simple semiring; the latter, in the notations introduced before Theorem 3.11, would imply that $\mathbf{F}_{M}=\operatorname{End}(M)$, what, by [33, Proposition 4.9 and Remark 4.10], would be a contradiction with that $M$ is a non-distributive lattice. Thus, there is no nonzero surjective homomorphism $\alpha: \operatorname{End}(M) \longrightarrow S$ to an ideal-simple semiring $S$, and therefore, $\mathcal{R}_{B M}(\operatorname{End}(M))=\operatorname{End}(M)$.

However, $F_{1}\left(i d_{M}\right)=\left\{\left(i d_{M} f+g i d_{M}, f+g\right) \mid f, g \in \operatorname{End}(M)\right\}=\triangle_{\operatorname{End}(M)}$. So, the congruence $\rho_{F_{1}\left(i d_{M}\right)}=\Delta_{E n d(M)}$, and therefore, $\left(i d_{M}, 0\right) \notin \rho_{F_{1}\left(i d_{M}\right)}$ and $\operatorname{End}(M)$ is not $F_{1}$-regular semiring.

In light of these observations, it seems to be natural to bring up the following problem.

Problem 2. Describe all hemirings for which the implication (i) $\Longrightarrow$ (ii) in Theorem 3.12 is true. 
Extending the corresponding result for rings (see, i.g., [4, Theore. 4.8.1]), in the next theorem we present an alternative, "working," description of the Brown-McCoy radical of hemirings from a fairly large class - including, in particular, all commutative hemirings and rings — of hemirings. To do that, we first note the following, generally useful and more or less obvious, fact.

Lemma 3.14. If $S$ is an ideal of a hemiring $R$ and $\rho$ is a congruence on $S$ such that $S / \rho$ is a semiring with the identity element $\bar{e}$, then the relation $\bar{\rho}$ on $R$, where $(a, b) \in \bar{\rho}$ iff (eae, ebe) $\in \rho$ for all $a, b \in R$, is a congruence on $R$ and $\rho \subseteq \bar{\rho}$. Furthermore, the natural map $\varphi: R / \bar{\rho} \longrightarrow S / \rho$ given by $\bar{r} \longmapsto \overline{\text { ere }}$ is a hemiring isomorphism; in particular, if $R$ is a semiring, then $\varphi$ is a semiring isomorphism.

Proof. It is clear that $\bar{\rho}$ is an equivalence relation on $R$. Now, let $(a, b) \in \bar{\rho}$ and $c \in R$. For $e \in S$ and $S$ is an ideal of $R$, one has $(e a e, e b e) \in \rho$ and ece $\in S$, and hence, $(e(a+c) e, e(b+c) e)=(e a e, e b e)+(e c e, e c e) \in \rho$; and therefore, $(a+c, b+c) \in \bar{\rho}$. Also, from $e c, a e \in S$ and (eae, ebe) $\in \rho$, we have $($ eceae, ecebe $) \in \rho$. Since $\bar{e}$ is an identity of $S / \rho$, one has $(e c e, e c) \in \rho$, and hence, $($ eceae, ecae $) \in \rho$. Similarly, one gets $($ ecebe, ecbe $) \in \rho$. Whence, (ecae, ecbe $) \in \rho$ and $(c a, c b) \in \bar{\rho}$. In the similar way, one can show that $(a c, b c) \in \bar{\rho}$, too. Thus, $\bar{\rho}$ is a congruence on $R$. The rest is trivial.

For any hemiring $R$, let $\mathfrak{C}_{s}$ stay for the set of all congruence $\rho$ on $R$ such that $R / \rho$ is a nonzero simple semiring, and $\rho_{s}:=\cap\left\{\rho \mid \rho \in \mathfrak{C}_{s}\right\}$. For each $\rho \in \mathfrak{C}_{s}$, by $\left[0_{\rho}\right]$ we denote the kernel of $\rho$, i.e., $\left[0_{\rho}\right]:=\{r \in R \mid$ $(r, 0) \in \rho\} \subseteq R$; and let $\mathcal{R}_{\mathfrak{C}_{s}}(R):=\cap\left\{\left[0_{\rho}\right] \mid \rho \in \mathfrak{C}_{s}\right\}$. It is easy to see that $\mathcal{R}_{\mathfrak{C}_{s}}(R)$ is a subtractive ideal of $R$, and $a \in \mathcal{R}_{\mathfrak{C}_{s}}(R)$ iff $(a, 0) \in \rho_{s}$, i.e., $\mathcal{R}_{\mathfrak{C}_{s}}(R)$ is the kernel of $\rho_{s}$.

Also, we say that a hemiring $R$ is strongly subtractive if every ideal $I$ of $R$ is a subtractive hemiring. The class of strongly subtractive hemirings, obviously, includes all rings, but not only them, namely ( $c f$. [15, Fact 2.1]): Let $R$ be the chain $0<a<b<1$ with the operation of multiplication defined as follows: $b^{2}=b, a^{2}=0, b a=a, a b=0$; then, $(R, \vee, \cdot)$ is a semiring and $\{0\},\{0, a\},\{0, a, b\}$ and $R$ are the only ideals of $R$, and they all are clearly subtractive hemirings.

Theorem 3.15. Let $R$ be a commutative (or strongly subtractive, or latticeordered) hemiring. Then, $\mathcal{R}_{B M}(R)=\mathcal{R}_{\mathfrak{C}_{s}}(R)$.

Proof. For each $\left[0_{\rho}\right], \rho \in \mathfrak{C}_{s}$, is a subtractive ideal of $R$ that is the kernel of the natural projection $\pi: R \longrightarrow R / \rho$ and [5, Proposition 10.16], $R /\left[0_{\rho}\right]$ and $R / \rho$ are semiisomorphic. As by [7, Theorem 2.6(c)] $\mathbb{S}=\{R \in$ $\left.\mathbb{H} \mid \mathcal{R}_{B M}(R)=0\right\}$ is a semisimple class of $\mathbb{H}$, and $\mathcal{R}_{B M}(R / \rho)=0$, i.e., 
$R / \rho \in \mathbb{S}$ since $R / \rho$ is a simple semiring, from [8, Theorem 3.7(b)] it follows that $R /\left[0_{\rho}\right] \in \mathbb{S}$, i.e., $\mathcal{R}_{B M}\left(R / 0_{\rho}\right)=0$, too. Whence, taking into consideration that by [25, Theorem 4.9] $\mathcal{R}_{B M}(R)=\cap\left\{K \in \mathcal{S} \mathcal{I}(R) \mid \mathcal{R}_{B M}(R / K)=\right.$ $0\}$, we have $\mathcal{R}_{B M}(R) \subseteq \cap\left\{0_{\rho} \mid \rho \in \mathfrak{C}_{s}\right\}=\mathcal{R}_{\mathfrak{C}_{s}}(R)$.

Now let $S$ stay for $\mathcal{R}_{\mathfrak{C}_{s}}(R)$. Using the A-D-S-property of a radical class (see, [25, Theorem 6.2]), we have $\mathcal{R}_{B M}(S) \subseteq \mathcal{R}_{B M}(R)$ for the ideal $\mathcal{R}_{B M}(S)$ of $R$. Suppose that $\mathcal{R}_{B M}(S) \neq S$. It is clear that $S$ is a commutative, or subtractive, hemiring as soon as $R$ is a commutative, or strongly subtractive, hemiring itself. Also, if $R$ is a lattice-ordered hemiring, $S$ is a lattice-ordered hemiring, too: Indeed, for any $a, b \in S$, we have $a+(a \wedge b)=a \vee(a \wedge b)=a$, and as $S$ is a subtractive ideal of $R$ and $a \in S$, we conclude that $a \wedge b \in S$. From these observations and Theorem 3.12, it follows that there exists an element $e \in S$ such that $e \notin \rho_{F_{1}(e)}$. For the Zorn's Lemma, let $\rho$ be a maximal congruence on $S$ such that $F_{1}(e) \subseteq \rho$ and $(e, 0) \notin \rho$. As in Theorem 3.12 , it can be shown that $S / \rho$ is a simple semiring with the identity $\bar{e} \in$ $S / \rho$ for $e \in S$. Applying Lemma 3.14, we have the congruence $\bar{\rho}$ on $R$ such that for all $a, b \in R$,

$$
(a, b) \in \bar{\rho} \Longleftrightarrow(e a e, e b e) \in \rho
$$

and $\rho \subseteq \bar{\rho}$. It is clear that $(e, 0) \notin \bar{\rho}$ and $(e x, x),(x e, x) \in \bar{\rho}$ for all $x \in R$; and hence, $F_{1}(e):=\{(e x+y e, x+y) \mid x, y \in R\} \subseteq \bar{\rho}$. Again, for the Zorn's Lemma, let $\delta$ be a maximal congruence on $R$ such that $F_{1}(e) \subseteq \delta$ and $(e, 0) \notin \delta$; and as in Theorem 3.12, we get that $R / \delta$ is a simple semiring with $(e, 0) \notin \delta$ that is a contradiction with $e \in \mathcal{R}_{\mathfrak{C}_{s}}(R)=S$. Therefore, $\mathcal{R}_{B M}(S)=S$ and $\mathcal{R}_{\mathfrak{C}_{s}}(R) \subseteq \mathcal{R}_{B M}(R)$.

Applying Theorem 3.15, we obtain the following complete descriptions of $\mathcal{R}_{B M}$-semisimple commutative, or lattice-ordered, hemirings.

Corollary 3.16. For a commutative hemiring $R$, the following conditions are equivalent:

(i) $\mathcal{R}_{B M}(R)=0$;

(ii) $\mathcal{R}_{\mathfrak{C}_{s}}(R)=0$;

(iii) $R$ is semiisomorphic to a subdirect product of simple commutative semirings;

(iv) $R$ is semiisomorphic to a subdirect product of semirings that are either fields or the semifield $\mathbf{B}$.

Proof. (i) $\Longleftrightarrow$ (ii) by Theorem 3.15 .

The equivalence (iii) $\Longleftrightarrow$ (iv) follows from the fact ([23, Theorem 3.2], or [1, Thorem 10.1]) that a simple commutative semiring is either a field or the semifield $\mathbf{B}$. 
(ii) $\Longrightarrow$ (iii). As $\mathcal{R}_{\mathfrak{C}_{s}}(R)=0$, one has that $R$ is semiisomorphic to a subdirect product $\prod_{\rho \in \mathfrak{C}_{s}}^{s u b} R / \rho$ of simple commutative semirings $R / \rho, \rho \in \mathfrak{C}_{s}$.

(iii) $\Longrightarrow$ (i). As simple semirings are obviously $\mathcal{R}_{B M}$-semisimple, the implication immediately follows from [8, Theorem 4.3 and Theorem 3.7 (b)].

Corollary 3.17. For a lattice-ordered hemiring $R$, the following conditions are equivalent:

(i) $\mathcal{R}_{B M}(R)=0$;

(ii) $\mathcal{R}_{\mathfrak{C}_{s}}(R)=0$;

(iii) $R$ is semiisomorphic to a subdirect product of simple lattice-ordered semirings;

(iv) $R$ is semiisomorphic to a subdirect product of the Boolean semifields B.

Proof. Using the fact (see, [16, Theorem 6.7]) that a lattice-ordered semiring $S$ is simple iff $S \cong \mathbf{B}$, this result can be proved in the similar fashion as in Corollary 3.16.

Let $\operatorname{End}(M)$ be an endomorphism semiring of a non-distributive finite lattice $M$. By [33, Proposition 2.3], this congruence-simple semiring is not commutative, not subtractive, and not lattice-ordered. Also, as was shown in Example 3.13, it has no nonzero surjective homomorphisms onto simple semirings, and therefore, $\mathcal{R}_{\mathfrak{C}_{s}}(\operatorname{End}(M))=\operatorname{End}(M)=\mathcal{R}_{B M}(\operatorname{End}(M))$. In light of this observation and Theorem 3.15, we conclude this section with the following, as we think interesting, question:

Problem 3. Is it true that $\mathcal{R}_{B M}(R)=\mathcal{R}_{\mathfrak{C}_{s}}(R)$ for all hemirings $R \in \mathbb{H}$ ? If the answer is "NO," then describe all hemirings $R \in \mathbb{H}$ for which $\mathcal{R}_{B M}(R)=$ $\mathcal{R}_{\mathfrak{C}_{s}}(R)$.

\section{The Nakayama's Lemma and Jacobson - Chevalley Density Theorem for Semirings}

In this section, we establish semiring analogs of the well-known classical ring results - Nakayama's and Hopkins Lemmas and Jacobson-Chevalley Density Theorem - by reducing our semiring settings to corresponding original ring ones. But to do that and for the reader's convenience, we first recall some notions we need in a sequel.

On any left semimodule $M$ over a hemiring $R$, there exists the congruence, "三", defined for all $m, m^{\prime} \in M$ as follows: $m \equiv m^{\prime}$ iff $m+x=m^{\prime}+x$ for some $x \in M$. Let $M^{*}$ and $m^{*} \in M^{*}$ stay for the factor semimodule 
$M / \equiv$ and equivalence class of an element $m \in M$, respectively. In particular, considering a hemiring $R$ as the regular semimodule ${ }_{R} R$, it is easy to see that $R^{*}$ becomes a hemiring with the multiplication $r^{*} s^{*}=(r s)^{*}$ for all $r^{*}, s^{*} \in R^{*}$. It is obvious that ${ }_{R} M \cong{ }_{R} M^{*}$ for any cancellative semimodule $M$, and $M^{*}$ is also a cancellative left $R^{*}$-semimodule with the scalar multiplication defined for all $m^{*} \in M^{*}$ and $r^{*} \in R^{*}$ by $r^{*} m^{*}=(r m)^{*}$. Furthermore, it is easy to see that $M^{*}$ is an irreducible left $R^{*}$-semimodule for any irreducible left $R$-semimodule $M$, and if $M$ is an irreducible left $R^{*}$ semimodule, then $M$ is also an irreducible left $R$-semimodule with the scalar multiplication given by $r m=r^{*} m$ for all $r \in R$ and $m \in M$. From these remarks and [9, Thorem 8], it follows that $J(R)=\left\{r \in R \mid r^{*} \in J\left(R^{*}\right)\right\}$ (see, also [9, p. 420]).

For any left $R$-semimodule $M$, there exists the left $R$-module of differences $D(M)$ of $M$ [5, Chapter 16] defined as the factor semimodule of the left $R$-semimodule $M \times M$ with respect to the subsemimodule $W=\{(m, m)$ $\mid m \in M\} \subseteq M \times M$, i.e., $D(M):=(M \times M) / W$. In fact, the semimodule $D(M)$ is a left $R$-module since for any $\left(m, m^{\prime}\right) \in M \times M$ in $D(M)$ one has $\overline{\left(m, m^{\prime}\right)}+\overline{\left(m^{\prime}, m\right)}=\overline{(0,0)}$. Also, there exists the canonical $R$ homomorphism $\xi_{M}: M \longrightarrow D(M)$ given by $m \longmapsto \overline{(m, 0)}$. In the case when $M$ is a cancellative semimodule, $\xi_{M}$ is injective, and therefore, we can consider the elements $\overline{(m, 0)}$ and $m$ to be the same and any element $\overline{\left(m, m^{\prime}\right)} \in D(M)$ to be the "difference" of the elements $\overline{(m, 0)}$ and $\overline{\left(0, m^{\prime}\right)}$, i.e., $D(M)=\left\{m-m^{\prime} \mid m, m^{\prime} \in M\right\}$. In particular, the left $R$-module of differences $D(R)$ of the regular semimodule ${ }_{R} R$ can be considered as a ring the ring of differences of $R$ [5, Chapter 8, p. 101] — with the operation of multiplication defined for all $a, b, c, d \in R$ by $\overline{(a, b)(c, d)}=\overline{(a c+b d, a d+c b)}$; and if $R$ is a semiring, then the ring of differences $D(R)$ is also a semiring with the identity $\overline{(1,0)}$. Moreover, it is easy to see that $D(M)$ becomes a left $D(R)$-module with $\overline{(a, b)} \overline{\left(m_{1}, m_{2}\right)}=\overline{\left(a m_{1}+b m_{2}, a m_{2}+b m_{1}\right)}$ for all $a, b \in$ $R$ and $m_{1}, m_{2} \in M$, and ${ }_{R} D(M) \cong{ }_{R} D\left(M^{*}\right)$, as well as $D(R) \cong D\left(R^{*}\right)$ as rings. Then, it is easy to see (see also [9, p. 419, Section 4 c)]) that a cancellative left $R$-semimodule $M$ is irreducible iff $D(M)$ is an irreducible left $D(R)$-module, as well as $J\left(R^{*}\right)=J\left(D\left(R^{*}\right)\right) \cap R^{*}=J(D(R)) \cap R^{*}$ for any hemiring $R[9$, p. 420, Section 4 e)].

Now we introduce the concept of a weakly-finitely (in short, $w$-finitely) generated semimodule, which is a very natural generalization of the notion of an irreducible semimodule and will prove to be useful in the "reduction procedure" from our semiring settings to corresponding ring ones in a sequel.

Definition 4.1. A left $R$-semimodule $M$ over a hemiring $R$ is called $w$ finitely generated if there exist a natural number $n$ and pairs $\left(m_{1}, m_{1}^{\prime}\right)$, $\left(m_{2}, m_{2}^{\prime}\right), \ldots,\left(m_{n}, m_{n}^{\prime}\right) \in M^{2}$ such that, for any element $m \in M$, there exist 
pairs $\left(r_{1}, s_{1}\right),\left(r_{2}, s_{2}\right), \ldots,\left(r_{n}, s_{n}\right) \in R^{2}$ and

$m+\left(r_{1} m_{1}^{\prime}+s_{1} m_{1}\right)+\ldots+\left(r_{n} m_{n}^{\prime}+s_{n} m_{n}\right)=\left(r_{1} m_{1}+s_{1} m_{1}^{\prime}\right)+\ldots+\left(r_{n} m_{n}+s_{n} m_{n}^{\prime}\right)$.

Proposition 4.2. (i) Any irreducible left $R$-semimodule is w-finitely generated as well.

(ii) Any finitely generated left $R$-semimodule is $w$-finitely generated as well.

(iii) For any $w$-finitely generated left $R$-semimodule $M$, the semimodule $M^{*}$ is an $w$-finitely generated left $R^{*}$-semimodule.

(iv) For any $w$-finitely generated left $R$-semimodule $M$, the left $D(R)$ module $D(M)$ is finitely generated.

(v) A cancellative left $R$-semimodule $M$ is w-finitely generated iff the left $D(R)$-module $D(M)$ is finitely generated.

Proof. (i), (ii) and (iii) are clear.

(iv). Let $M$ be an $w$-finitely generated left $R$-semimodule, and $\left(m_{1}, m_{1}^{\prime}\right),\left(m_{2}, m_{2}^{\prime}\right), \ldots\left(m_{n}, m_{n}^{\prime}\right) \in M^{2}$ be the pairs as in Definition 4.1. Then one may easily see that $D(M)=D(R) \overline{\left(m_{1}, m_{1}^{\prime}\right)}+\ldots+D(R) \overline{\left(m_{n}, m_{n}^{\prime}\right)}$, and therefore, $D(M)$ is a finitely generated left $D(R)$-module.

(v). This follows from (iv) and the fact that $D(M)=\left\{m-m^{\prime} \mid m, m^{\prime} \in\right.$ $M\}$ when $M$ is cancellative.

As usually (see, e.g., [5, pages 50, 155]), $Z(R)=\{r \in R \mid r+x=x$ for some $x \in R\}$ and $Z(M)=\{m \in M \mid m+x=x$ for some $x \in M\}$ denote the zeroids of a hemiring $R$ and an $R$-semimodule $M$, respectively. We are now ready to present a semiring version of the fundamental in the theory of rings and modules, famous Nakayama's Lemma (see, for instance, [18, Nakayama's Lemma 4.22]).

Theorem 4.3. Let $R$ be a semiring with $1 \notin Z(R)$, and $I$ a left ideal of $R$. Then, the following statements are equivalent:

(i) $I \subseteq J(R)$;

(ii) For any $w$-finitely generated left $R$-semimodule $M$, if $I M=M$, then $M=Z(M)$.

Proof. Because $1 \notin Z(R)$, we first note that $R^{*}$ and $D\left(R^{*}\right)$ are nonzero additively cancellative semiring and ring, respectively, with the identity ${ }^{*}$.

(i) $\Longrightarrow$ (ii). Let $I M=M$ for a left ideal $I \subseteq J(R) \subseteq R$ of a semiring $R$ and an $w$-finitely generated left $R$-semimodule $M$, and $I^{*}:=\left\{r^{*} \mid r \in I\right\} \subseteq$ $R^{*}$. It is clear that $I^{*}$ is a left ideal of $R^{*}$ and $I^{*} \subseteq J\left(R^{*}\right)$ since by [9, p. 420 , Section $4 \mathrm{e})] J(R)=\left\{r \mid r^{*} \in J\left(R^{*}\right)\right\}$. Also, it is clear that $D\left(I^{*}\right):=$ $\left\{r^{*}-s^{*} \mid r^{*}, s^{*} \in I^{*}\right\}$ is a left ideal of $D\left(R^{*}\right)$, and even $D\left(I^{*}\right) \subseteq J\left(D\left(R^{*}\right)\right)$ because of $I^{*} \subseteq J\left(R^{*}\right)$ and $J\left(D\left(R^{*}\right)\right)$ is an ideal of $D\left(R^{*}\right)$, and, by [9, p. 
420, Section $4 \mathrm{e})], J\left(R^{*}\right)=J\left(D\left(R^{*}\right)\right) \cap R^{*}$. From $I M=M$, it follows that $I^{*} M^{*}=M^{*}$ and, hence, $D\left(I^{*}\right) D\left(M^{*}\right)=D\left(M^{*}\right)$. Applying Proposition 4.2 (iii) and (iv), one has that $D\left(M^{*}\right)$ is a finitely generated left $D\left(R^{*}\right)$-module. From these observations and using the Nakayama's Lemma for rings (see, for example, [18, Nakayama's Lemma 4.22]), we have $D\left(M^{*}\right)=0$; hence, $M^{*}=0$, and therefore, $M=Z(M)$.

(ii) $\Longrightarrow(\mathrm{i})$. In the notations introduced in the previous implication and assuming that $I \nsubseteq J(R)$, we shall show that $D\left(I^{*}\right) \nsubseteq J\left(D\left(R^{*}\right)\right)$ : Indeed, if $D\left(I^{*}\right) \subseteq J\left(D\left(R^{*}\right)\right)$, then $I^{*} \subseteq J\left(D\left(R^{*}\right)\right)$ and, by [9, p. 420, Section $\left.\left.4 \mathrm{e}\right)\right]$, $I^{*} \subseteq R^{*} \cap J\left(D\left(R^{*}\right)\right)=J\left(R^{*}\right)$; whence, using that by [9, p. 420, Section 4 e)] $J(R)=\left\{r \mid r^{*} \in J\left(R^{*}\right)\right\}$, we have that $I \subseteq J(R)$. Thus, there exists an irreducible left $D\left(R^{*}\right)$-module $M$ and $D\left(I^{*}\right) M \neq 0$. As $M$ is irreducible, $D\left(I^{*}\right) M=M$, and since $(M,+)$ is a group, we also have $I^{*} M=D\left(I^{*}\right) M=$ $M$. For $M$ is an irreducible left $D\left(R^{*}\right)$-module, we have that $M$ is an irreducible left $R$-semimodule with respect to the multiplication defined by $r m=r^{*} m$ for all $r \in R$ and $m \in M$. Then, applying Proposition 4.2 (i), we have $M$ is a nonzero $w$-finitely generated left $R$-semimodule with $I M=I^{*} M=M$, but $M \neq 0=Z(M)$. Therefore, $I \subseteq J(R)$.

As a corollary of Theorem 4.3, we obtain the following semiring version of the Hopkins's Theorem for rings (see, e.g., [4, Lemma 4.5.8], or [18, Theorem 4.12]) .

Corollary 4.4. Let $R$ be a left artinian semiring. Then, there exists a natural number $n \in \mathbb{N}$ such that $J(R)^{n}=Z(R)$.

Proof. First note that for any irreducible $R$-semimodule $M$ and $z \in Z(R)$, always $z \in(0: M)_{R}$ : Indeed, if $z+x=x$ for some $x \in M$, then $z m+x m=$ $x m$ for any $m \in M$, and hence, $z m=0$ and $z \in(0: M)_{R}$. Whence, $Z(R) \subseteq J(R)$. Therefore, if $1 \in Z(R)$, then it is clear that $R=Z(R)$, and hence, $J(R)^{n}=Z(R)$ for any $n \in \mathbb{N}$.

Now let $1 \notin Z(R)$. For $R$ is a left artinian semiring, $R^{*}$ is also left artinian semiring; hence, in the filtration

$$
J\left(R^{*}\right) \supseteq J\left(R^{*}\right)^{2} \supseteq J\left(R^{*}\right)^{3} \supseteq \cdots,
$$

$J\left(R^{*}\right)^{n}=J\left(R^{*}\right)^{n+1}$ for some positive $n \in \mathbb{N}$. If $J\left(R^{*}\right)^{n} \neq 0$, then the set of all left ideals $I$ of $R^{*}$ for which $J\left(R^{*}\right)^{n} I \neq 0$ is not empty. And therefore, by [13, Proposition 2.1], there exists a minimal left $I$ ideal of $R^{*}$ with $J\left(R^{*}\right)^{n} I \neq 0$. Let $x \in I$ and $J\left(R^{*}\right)^{n} x \neq 0$. Then, for $J\left(R^{*}\right) x \subseteq R^{*} x \subseteq I$ and $J\left(R^{*}\right)^{n}\left(J\left(R^{*}\right) x\right)=J\left(R^{*}\right)^{n+1} x=J\left(R^{*}\right)^{n} x \neq 0$, one has $J\left(R^{*}\right) x=I=$ $R^{*} x$, and hence, by Theorem 4.3,J(R*) $\left(R^{*} x\right)=R^{*} x=I=0$. Thus, $J\left(R^{*}\right)^{n}=0$, and therefore, $J(R)^{n}=Z(R)$ since by [9, p. 420, Section 4 e)] $J(R)=\left\{r \mid r^{*} \in J\left(R^{*}\right)\right\}$. 
Recall (see, e.g., [18, Chapter 4]), a subring $R \subseteq \operatorname{End}\left({ }_{D} M\right)$ of the endomorphism ring $\operatorname{End}\left({ }_{D} M\right)$ of a left vector space ${ }_{D} M$ over a division ring $D$ is said to be dense if, for any linearly independent elements $m_{1}, \ldots, m_{n} \in M$ and any elements $m_{1}^{\prime}, \ldots, m_{n}^{\prime} \in M$, there exists $r \in R$ such that $m_{i} r=m_{i}^{\prime}$ for $i=1,2, \ldots, n$. Our next result is a hemiring analog of the JacobsonChevalley Density Theorem for primitive rings (see, for instance, 44, Theorem 4.5.3]).

Theorem 4.5. A hemiring $R$ is primitive iff it is semiisomorphic to an additively cancellative hemiring $S$ whose ring of differences $D(S)$ is, in turn, isomorphic to a dense subring of linear transformations of a vector space over a division ring.

Proof. $\Longrightarrow$. Let $M$ be a faithful irreducible left $R$-semimodule $M$, and $\varphi: R \longrightarrow \operatorname{End}(M,+)$ the natural hemiring homomorphism such that $\varphi(r)(m)=r m$ for all $r \in R$ and $m \in M$ and, hence, having $\operatorname{ker}(\varphi):=$ $\varphi^{-1}(0)=(0: M)_{R}=0$. So, there exist the natural congruence $\equiv_{\varphi}$ on $R$ induced by $\varphi$, the natural surjection $\pi: R \longrightarrow R / \equiv_{\varphi}$ with $\operatorname{ker}(\pi):=$ $\pi^{-1}(\overline{0})=(0: M)_{R}=0$, i.e., $\pi$ is a semiisomorphism, and the natural injection $\psi: R / \equiv_{\varphi} \longrightarrow \operatorname{End}(M,+)$ such that $\psi(\bar{r})=\varphi(r)$ for all $r \in R$. For $(M,+)$ is additively cancellative, both $\operatorname{End}(M,+)$ and $R / \equiv_{\varphi}$ are additively cancellative hemirings, too. If $S$ stays for the hemiring $R / \equiv_{\varphi}$, $M$ becomes an irreducible left $S$-semimodule ${ }_{S} M$ with $\bar{r} m=\mathrm{rm}$ for all $m \in M$ and $r \in R$. Then, $D(M)$ is an irreducible left $D(S)$-module with $(0: D(M))_{D(S)}=\{\bar{r}-\bar{s} \mid(\bar{r}-\bar{s}) m=0$ for all $m \in M\}=\{\bar{r}-\bar{s} \mid r m=s m$ for all $m \in M\}=\left\{\bar{r}-\bar{s} \mid r \equiv_{\varphi} s\right\}=0$. Whence it follows that $D(S)$ is a primitive ring. Applying [4, Theorem 4.5.3], we immediately obtain that $D(S)$ is isomorphic to a dense subring of linear transformations of a space over a division ring.

$\Longleftarrow$. Let $\pi: R \longrightarrow S$ be a semiisomorphism to an additively cancellative hemiring $S$ with $D(S)$ to be isomorphic to a dense subring of linear transformations of a vector space over a division ring. By [4, Theorem 4.5.3], $D(S)$ is a primitive ring, and therefore, there exists a faithful irreducible left $D(S)$-module $M$ which is also a faithful irreducible left $S$-semimodule. Then $M$ is obviously a left $R$-semimodule with $r m=\pi(r) m$ for all $r \in R$ and $m \in M$; and $(0: M)_{R}=\{r \in R \mid \pi(r) m=0$ for all $m \in M\}=\{r$ $\left.\mid \pi(r) \in(0: M)_{S}=0\right\}=\operatorname{ker}(\pi)=0$. For ${ }_{S} M$ is irreducible, ${ }_{R} M$ is also irreducible and, therefore, $R$ is a primitive hemiring.

Combining Theorem 4.5 and Corollary 3.8, one obtains

Corollary 4.6. A hemiring $R$ is J-semisimple iff it is semiisomorphic to a subdirect product of some additively cancellative hemirings $S$ whose rings of 
differences $D(S)$ are isomorphic to dense subrings of linear transformations of vector spaces over division rings.

A right congruence on a hemiring $R$ is just a congruence on the (right) regular semimodule $R_{R}$. And we say that a hemiring $R$ is (right) congruenceartinian iff the descending chain condition on right congruences on $R$ is held. Our next observation, a corollary of Theorem 4.5, is a hemiring version of the classical structure theorem for artinian primitive ring (see, e.g., [4, Proposition 4.5.4], or [18, Theorem 4.11.19]):

Corollary 4.7. A primitive congruence-artinian hemiring $R$ is semiisomorphic to an additively cancellative hemiring $S$ whose ring of differences $D(S)$, in turn, is isomorphic to a full ring of linear transformations on a finite dimensional vector space over a division ring.

Proof. By Theorem 4.5, there exists a semiisomorphism $f: R \longrightarrow S$ to an additively cancellative hemirings $S$ such that $D(S)$ is isomorphic to a dense subring of linear transformations $\operatorname{End}\left({ }_{F} V\right)$ of a vector space $V$ over a division $\operatorname{ring} F$. We shall show that $\operatorname{dim}\left({ }_{F} V\right)<\infty$. Indeed, suppose that $\left\{e_{1}, e_{2}, \ldots, e_{n}, \ldots\right\}$ is a basis of ${ }_{F} V$ and consider the subspaces $V_{n}$ generated by $e_{1}, \ldots, e_{n}$ for $n=1,2, \ldots$. Obviously, all $\left(0: V_{n}\right)_{D(S)}$ are right ideals of $D(S)$, and

$$
\left(0: V_{1}\right)_{D(S)} \supset\left(0: V_{2}\right)_{D(S)} \supset \ldots \supset\left(0: V_{n}\right)_{D(S)} \supset \ldots
$$

is a strictly descending chain according to [4, Lemma 4.5.2]. It is clear that $\left(0: V_{n}\right)_{D(S)}=\left\{s-s^{\prime} \in D(S) \mid s, s^{\prime} \in S \& \forall x \in V_{n}\left(x s=x s^{\prime}\right)\right\}$ and $\alpha_{n}:=\left\{\left(s, s^{\prime}\right) \mid s, s^{\prime} \in S \& \forall x \in V_{n}\left(x s=x s^{\prime}\right)\right\}, n=1,2, \ldots$, are a right congruences on $S$. Therefore, there is a strictly descending chain of right congruences $\alpha_{n}$ :

$$
\alpha_{1} \supset \alpha_{2} \supset \ldots \supset \alpha_{n} \supset \ldots
$$

So, there is the strictly descending chain of right congruences $\beta_{n}:=\left\{\left(r, r^{\prime}\right)\right.$ | $\left.\left(f(r), f\left(r^{\prime}\right)\right) \in \alpha_{n}\right\}, n=1,2, \ldots$, on a congruence-artinian hemiring $R$. Thus, $\operatorname{dim}\left({ }_{F} V\right)<\infty$, and therefore, $D(S)=\operatorname{End}\left({ }_{F} V\right)$.

As was mentioned earlier and in connection with Problem 1, we conclude this section with the following observation:

Proposition 4.8. $J_{s}(R) \subseteq J(R)$ for any commutative or additively regular, in particular additively idempotent, hemiring $R$.

Proof. Let $R$ be a commutative hemiring. By [25, Theorem 4.9], $J(R)=$ $\cap\{I \in \mathcal{S I}(R) \mid J(R / I)=0\}$, where every commutative $J$-semisimple hemiring $R / I$ is, by Corollary 3.8, semiisomorphic to a subdirect product of commutative primitive hemirings. Taking into consideration Theorem 4.5, we 
note that a commutative hemiring $S$ is primitive iff it is semiisomorphic to an additively cancellative commutative hemiring $H$ whose ring of differences $D(H)$ is a field. If the hemiring $H$ is itself a ring, then it is a field and $J_{s}(H)=J(H)=0$. Otherwise, we shall show that $H$ is semiisomorphic to the semifield $\mathbf{B}$.

First, $H$ is zerosumfree: Indeed, if $a+b=0$ for some $a, b \in H$ and $a \neq 0$, then there exist $x, y \in H$ such that in the field of differences $D(H)$ we have $a(x-y)=1$, that is, $a x=1+a y$ and, hence, $0=a x+b x=1+(a y+b x)$, what implies a contradiction with that $H$ is not a ring. Secondly, $H$ is entire since it is a cancellative hemiring and therefore is a subhemiring of the field $D(H)$. Thus, we have the semiisomorphism $f: H \longrightarrow \mathbf{B}$ given by $h \longmapsto 1$ for all nonzero $h \in H$. As was mentioned in Example 3.7, $J_{s}(\mathbf{B})=0$, and therefore, by [8, Theorem $3.7(\mathrm{~b})], J_{s}(H)=0$, too.

From these observations and using [8, Theorem 3.7 (b)] one more time, we conclude that $J_{s}(R / I)=0$ for every commutative $J$-semisimple hemiring $R / I$. Then, applying [25, Theorem 4.9] again, we obtain the needed inclusion:

$$
\begin{aligned}
J(R) & =\cap\{I \in \mathcal{S I}(R) \mid J(R / I)=0\} \supseteq \\
\cap\{I \quad & \left.\in \mathcal{S I}(R) \mid J_{s}(R / I)=0\right\}=J_{s}(R) .
\end{aligned}
$$

The statement for additively regular hemirings can be established in the similar way by using Corollary 3.9 and the obvious fact that $J(R)=J_{s}(R)$ for rings $R$.

\section{Radicals and Morita Equivalence of Semir- ings}

Following [12] and [14, theorem 4.12], two semirings $R$ and $S$ are said to be Morita equivalent if the semimodule categories ${ }_{R} \mathcal{M}$ and ${ }_{S} \mathcal{M}$ are equivalent, i.e., there exist two additive functors $F:{ }_{R} \mathcal{M} \longrightarrow{ }_{S} \mathcal{M}$ and $G:{ }_{S} \mathcal{M} \longrightarrow{ }_{R} \mathcal{M}$ and natural isomorphisms $\eta: G F \longrightarrow I d_{R} \mathcal{M}$ and $\xi: F G \longrightarrow I d_{S} \mathcal{M}$. The semirings $R$ and $S$ are Morita equivalent iff the semimodule categories $\mathcal{M}_{R}$ and $\mathcal{M}_{S}$ are also equivalent [14, Theorem 4. 12]. Recall [14], a left semimodule $\left.{ }_{R} P \in\right|_{R} \mathcal{M} \mid$ is a generator for the category of left semimodules ${ }_{R} \mathcal{M}$ if the regular semimodule $\left.{ }_{R} R \in\right|_{R} \mathcal{M} \mid$ is a retract of a finite direct sum $\oplus_{i} P$ of the semimodule ${ }_{R} P$; and a finitely generated projective semimodule $\left.{ }_{R} P \in\right|_{R} \mathcal{M} \mid$ is called a progenerator for the category ${ }_{R} \mathcal{M}$ if it is generator for ${ }_{R} \mathcal{M}$. Also, a left semimodule $\left.{ }_{R} P \in\right|_{R} \mathcal{M} \mid$ is a progenerator for the category of left semimodules ${ }_{R} \mathcal{M}$ iff its trace ideal $\operatorname{tr}(P):=\sum_{f \in{ }_{R} \mathcal{M}\left({ }_{R} P,{ }_{R} R\right)} f(P)$ coincides with $R$, i.e., $\operatorname{tr}(P)=R$ [14, Proposition 3.9]. Then, the semirings $R$ 
and $S$ are Morita equivalent iff there exists a progenerator ${ }_{R} P \in\left|{ }_{R} \mathcal{M}\right|$ for ${ }_{R} \mathcal{M}$ such that the semiring $S$ and the endomorphism semiring $\operatorname{End}\left({ }_{R} P\right)$ are isomorphic semirings [14, Definition 4.1 and Theorem 4.12]. For the reader's convenience, we also reproduce here Proposition 5.2 of [16] that will prove to be useful in a sequence:

Proposition 5.1. (cf. [17, Proposition 18.33]) For semirings $R$ and $S$, the followings statements are equivalent:

(i) $R$ is Morita equivalent to $S$;

(ii) $S \cong e M_{n}(R)$ e for some idempotent $e$ in a matrix semiring $M_{n}(R)$ such that $M_{n}(R) e M_{n}(R)=M_{n}(R)$.

Proof. (i) $\Longrightarrow$ (ii). By [14, Theorem 4.12], there exists a progenerator ${ }_{R} P \in\left|{ }_{R} \mathcal{M}\right|$ for ${ }_{R} \mathcal{M}$ such that $S \cong \operatorname{End}\left({ }_{R} P\right)$ as semirings. Applying [14, Proposition 3.1] and without loss of generality, we can assume that the semimodule ${ }_{R} P$ is a subsemimodule of a free semimodule ${ }_{R} R^{n}$, and there exists an endomorphism $e \in \operatorname{End}\left({ }_{R} R^{n}\right)$ such that $e^{2}=e, P=e\left(R^{n}\right)$ and $\left.e\right|_{P}=i d_{P}$. For $e \in \operatorname{End}\left({ }_{R} R^{n}\right) \cong M_{n}(R)$, one can consider $e$ to be a right multiplication by some idempotent matrix $\left(a_{i j}\right) \in M_{n}(R)$. In the same fashion as it has been done in the case of modules over rings (see, for example, [17, Remark 18.10(D) and Exercise 2.18]), we obtain that $\operatorname{tr}(P)=$ $\sum R a_{i j} R$ and $r E_{i j} e E_{k l} r^{\prime}=r a_{j k} r^{\prime} E_{i l}$, where $\left\{E_{i j}\right\}$ are the matrix units in $M_{n}(R)$ and $r, r^{\prime} \in R$, and get that $M_{n}(R) e M_{n}(R)=M_{n}(t r(R))$. For ${ }_{R} P$ is a progenerator of the category of semimodules ${ }_{R} \mathcal{M}$ and [14, Proposition 3.9], $\operatorname{tr}(P)=R$ and, hence, $M_{n}(R) e M_{n}(R)=M_{n}(R)$. The proof of the implication is now completed by noting that the semiring homomorphism

$$
\theta: \operatorname{End}\left({ }_{R} P\right) \longrightarrow e \operatorname{End}\left({ }_{R} R^{n}\right) e \cong e M_{n}(R) e
$$

defined for all $f \in \operatorname{End}\left({ }_{R} P\right.$ by $f \longmapsto e i f e$, where $i: P \longrightarrow R^{n}$ is the natural injection, is a semiring isomorphism.

(ii) $\Longrightarrow(\mathrm{i})$. Let $S \cong e M_{n}(R) e$ for some idempotent $e$ of a matrix semiring $M_{n}(R)$ and $M_{n}(R) e M_{n}(R)=M_{n}(R)$. Then, modifying for semimodules the well-known for modules over rings results (see, for example, [18, Proposition 21.6 and Corollary 21.7]), one has $S \cong e M_{n}(R) e \cong$ $\operatorname{End}\left(M_{n}(R) M_{n}(R) e\right)$ with $M_{n}(R) e$ to be, by [14, Corollary 3.3], a projective left $M_{n}(R)$-semimodule. Moreover, for $\operatorname{tr}\left(M_{n}(R) M_{n}(R) e\right)=M_{n}(R) e M_{n}(R)$ $=M_{n}(R)$, the semimodule $M_{n}(R) M_{n}(R) e$ is a progenerator of the category of semimodules $M_{n}(R) \mathcal{M}$. From these observations, it follows that $S$ and $M_{n}(R)$ are Morita equivalent semirings, and therefore, applying [12, Theorem 5.14] and [14, Corollary 4.4], we conclude that the semirings $R$ and $S$ are Morita equivalent too. 
A semiring $R$ is zeroic [5, p. 50] if $Z(R)=R$, i.e., $1+r=r$ for some $r \in R$. From Proposition 5.1, it immediately follows that to be zeroic is a Morita property for semirings, namely:

Corollary 5.2. Let a zeroic semiring $R$ be Morita equivalent to a semiring $S$. Then, $S$ is also a zeroic semiring.

Proof. It is clear that a matrix semiring $M_{n}(R)$ is zeroic and, hence, the statement follows from Proposition 5.1 right away.

Let $D\left(\mathcal{M}_{R}\right)$ denote the full subcategory of $R$-modules of the semimodule category $\mathcal{M}_{R}$ over a semiring $R$ with an $R$-semimodule $M \in\left|D\left(\mathcal{M}_{R}\right)\right|$ iff the monoid $(M,+, 0)$ is an abelian group. For a nonzeroic semiring $R$, it is easy to see (see also [5, Section 16, p. 183]) that the category $D\left(\mathcal{M}_{R}\right)$ actually coincides with the category $\mathcal{M}_{D(R)}$ of right modules over the ring of differences $D(R)$.

Proposition 5.3. Let $F: \mathcal{M}_{R} \rightleftarrows \mathcal{M}_{S}: G$ be an equivalence of the semimodule categories $\mathcal{M}_{R}$ and $\mathcal{M}_{S}$ over nonzeroic semirings $R$ and $S$, respectively. Then, for the same functors $F$ and $G$, the following statements are true:

(i) $F: D\left(\mathcal{M}_{R}\right) \rightleftarrows D\left(\mathcal{M}_{S}\right): G$ is an equivalence of the modules categories $D\left(\mathcal{M}_{R}\right)$ and $D\left(\mathcal{M}_{S}\right)$ over the semirings $R$ and $S$, respectively;

(ii) $F: \mathcal{M}_{D(R)} \rightleftarrows \mathcal{M}_{D(S)}: G$ is an equivalence of the modules categories $\mathcal{M}_{D(R)}$ and $\mathcal{M}_{D(S)}$ over the rings of differences $D(R)$ and $D(S)$, respectively;

(iii) For any semimodules $M \in\left|\mathcal{M}_{R}\right|$ and $N \in\left|\mathcal{M}_{S}\right|$, there are semimodule isomorphisms of the modules of differences $F(D(M))_{S} \cong D(F(M))_{S}$ and $G(D(N))_{R} \cong D(G(N))_{R}$; in other words, the functors $F$ and $G$ preserve modules of differences;

(iv) The functors $F$ and $G$ preserve the classes of cancellative semimodules.

Proof. (i). This statement follows straightforwardly noting that by $[14$, Theorems 4.5 and 4.12] the functor $F$, for example, is isomorphic to additive functors $-\otimes_{R} P$ for a suitable bisemimodule ${ }_{R} P_{S}$.

(ii). It follows from item (i) and the remark above.

(iii). Let $M \in\left|\mathcal{M}_{R}\right|$. Then, using [5, Proposition 16.1], it is easy to see that the $R$-module of differences $D(M)$ is a colimit of some small diagram of semimodule surjections $\left\{M \rightarrow X|X \in| D\left(\mathcal{M}_{R}\right) \mid\right\}$. By [22, Theorem 5.5.1 and its dual], the functor $F$ preserves colimits and, therefore, now our assertions follows from (i).

(iv). As was mentioned in [5, p. 181], a semimodule $M$ is cancellative iff the canonical homomorphism $\xi_{M}: M \longrightarrow D(M)$ given by $m \longmapsto \overline{(m, 0)}$ 
(see Section 4) is injective. From this observation, dual of [14, Lemma 4.7] and (iii), we immediately deduce the statements.

We reformulate item (ii) of Proposition 5.3. as

Corollary 5.4. The Morita equivalence of nonzeroic semirings $R$ and $S$ implies the Morita equivalence of their rings of differences $D(R)$ and $D(S)$.

However, as the following obvious counterexample shows the inverse of this statement, in general, is not true.

Example 5.5. The ring of integers $\mathbb{Z}$ and the semiring $\mathbb{N}$ are clearly not Morita equivalent semirings, but $D(\mathbb{N})=D(\mathbb{Z})=\mathbb{Z}$.

Our next observation is that the classes of simple, semi-irreducible, and irreducible, semimodules are also "preserved" by Morita equivalences of semirings, more precisely:

Proposition 5.6. Let $F: \mathcal{M}_{R} \rightleftarrows \mathcal{M}_{S}: G$ be an equivalence of the semimodule categories $\mathcal{M}_{R}$ and $\mathcal{M}_{S}$ over semirings $R$ and $S$, respectively. Then, a semimodule $M \in\left|\mathcal{M}_{R}\right|$ is simple (semi-irreducible, irreducible) iff $F(M) \in\left|\mathcal{M}_{S}\right|$ is simple (semi-irreducible, irreducible).

Proof. First, note the following obvious facts for a nonzero semimodule $M \in\left|\mathcal{M}_{R}\right|:$ a) $M$ has no nonzero subsemimodules iff every nonzero homomorphism $f: L \longrightarrow M$ in $\mathcal{M}_{R}$ is a surjection; b) $M$ is congruence-simple iff every nonzero homomorphism $g: M \longrightarrow N$ in $\mathcal{M}_{R}$ is an injection; c) a cancellative semimodule $M$ is semi-irreducible iff $\operatorname{Ker}(f)=0$ for every nonzero homomorphism $f: M \longrightarrow N$ in $\mathcal{M}_{R}$.

Now, let $M \in\left|\mathcal{M}_{R}\right|$ be a simple semimodule, and $f: L \longrightarrow F(M)$ a nonzero homomorphism in $\mathcal{M}_{S}$. Then, $G(f): G(L) \longrightarrow G(F(M)) \cong M$ is also a nonzero homomorphism in $\mathcal{M}_{R}$, and therefore, it is a surjection. Whence by [14, Lemma 4.7], $F(G(f)): F(G(L)) \longrightarrow F(G(F(M))) \cong F(M)$ and, hence, $f$ are also surjections and $F(M)$ has no nonzero subsemimodules. For a nonzero homomorphism $g: F(M) \longrightarrow N$ in $\mathcal{M}_{S}$, we have $G(g): M \cong G(F(M)) \longrightarrow G(N)$ is a nonzero homomorphism in $\mathcal{M}_{R}$, and hence, it is injective. Then, applying the functor $F$ and dual of [14, Lemma 4.7], we have that $F(G(g)): F(M) \cong F(G(F(M))) \longrightarrow F(G(N)) \cong N$ is an injection and, hence, $g$ is also an injection. Thus, we have shown that $F(M)$ is a simple semimodule too.

Let $M \in\left|\mathcal{M}_{R}\right|$ be a semi-irreducible semimodule, and $g: F(M) \longrightarrow N$ a nonzero homomorphism in $\mathcal{M}_{S}$. Then, $G(g): M \cong G(F(M)) \longrightarrow G(N)$ is a nonzero homomorphism in $\mathcal{M}_{R}$ with $\operatorname{Ker}(G(g))=0$. Again, it is easy to see that $\operatorname{Ker}(G(g))$ is a limit of some small diagram of semimodule injections $\left\{i: X \longmapsto G(F(M))|X \in| \mathcal{M}_{R} \mid \& G(g) i=0\right\}$, and therefore, applying 
[22, Theorem 5.5.1 ], we have $\operatorname{Ker}(F(G(g)): F(M) \cong F(G(F(M))) \longrightarrow$ $F(G(N))) \cong N)=0$, and hence, $\operatorname{Ker}(g)=0$ and $F(M) \in\left|\mathcal{M}_{S}\right|$ is a semi-irreducible semimodule.

Finally, consider the case when a semimodule $M \in\left|\mathcal{M}_{R}\right|$ is irreducible. By Corollary 5.2, $R$ and $S$ are zeroic semirings simultaneously; and, clearly, over zeroic semirings there are no irreducible semimodules. Hence, for zeroic semirings $R$ and $S$ our assertion is true. So, assume that the semirings $R$ and $S$ are not zeroic. As was mentioned earlier, $M \in\left|\mathcal{M}_{R}\right|$ is irreducible iff $D(M) \in\left|\mathcal{M}_{D(R)}\right|$ is irreducible, and, applying Proposition 5.3, iff $F(D(M)) \in\left|\mathcal{M}_{D(S)}\right|$ is irreducible, iff $D(F(M)) \in\left|\mathcal{M}_{D(S)}\right|$ is irreducible and, finally, iff $F(M) \in\left|\mathcal{M}_{S}\right|$ is irreducible.

In our next result, we establish the fundamental relationship between the radicals $J, J_{s}$, and $\mathcal{R}_{B M}$ of semirings $R$ and matrix semirings $M_{n}(R)$, $n \geq 1$. But first note the following fact:

Lemma 5.7. A semiring $R$ is $\mathcal{R}_{B M}$-semisimple iff all matrix semirings $M_{n}(R), n \geq 1$, are $\mathcal{R}_{B M}$-semisimple, i.e., $\mathcal{R}_{B M}(R)=0$ iff $\mathcal{R}_{B M}\left(M_{n}(R)\right)=$ 0 for all $n \geq 1$.

Proof. In the same way as for matrix rings over rings, it is easy to show that any ideal of a matrix semiring $M_{n}(R)$ over a semiring $R$ consists of all matrices having all entries in some ideal of $R$. Hence, for a nonzero ideal $A$ of the semiring $M_{n}(R)$ there exists a nonzero ideal $I$ of a semiring $R$ such that $A=M_{n}(I)$. If the semiring $R$ is $\mathcal{R}_{B M}$-semisimple, i.e., $\mathcal{R}_{B M}(R)=0$, then $I \nsubseteq \mathcal{R}_{B M}(R)$ and there exists a nonzero surjective semiring homomorphism $f$ from $I$ to an ideal-simple semiring $S$. Whence, there exists a nonzero surjective homomorphism from $A=M_{n}(I)$ to the matrix semiring $M_{n}(S)$ that, by [15, Proposition 4.7], is also an ideal-simple semiring. Therefore, $\mathcal{R}_{B M}\left(M_{n}(R)\right)=0$.

Theorem 5.8. For all matrix semirings $M_{n}(R), n \geq 1$, over a semiring $R$, the following equations are held:

(i) $\mathcal{R}_{B M}\left(M_{n}(R)\right)=M_{n}\left(\mathcal{R}_{B M}(R)\right)$;

(ii) $J_{s}\left(M_{n}(R)\right)=M_{n}\left(J_{s}(R)\right)$;

(iii) $J\left(M_{n}(R)\right)=M_{n}(J(R))$.

Proof. (i). For $\mathcal{R}_{B M}(R)$ is a subtractive ideal of $R$, it follows that $M_{n}\left(\mathcal{R}_{B M}(R)\right)$ is a subtractive ideal of $M_{n}(R)$ and, hence,

$M_{n}(R) / M_{n}\left(\mathcal{R}_{B M}(R)\right) \cong M_{n}\left(R / \mathcal{R}_{B M}(R)\right)$. So, because of Lemma 5.2 and $\mathcal{R}_{B M}\left(R / \mathcal{R}_{B M}(R)\right)=0$, we get $\mathcal{R}_{B M}\left(M_{n}(R) / M_{n}\left(\mathcal{R}_{B M}(R)\right)\right)=$ $\mathcal{R}_{B M}\left(M_{n}\left(R / \mathcal{R}_{B M}(R)\right)\right)=0$. From the latter and since by [25, Theorem 4.9] $\mathcal{R}_{B M}\left(M_{n}(R)\right)=\cap\left\{K \in \mathcal{S} \mathcal{I}\left(M_{n}(R)\right) \mid \mathcal{R}_{B M}\left(M_{n}(R) / K\right)=0\right\} \subseteq$ $M_{n}\left(\mathcal{R}_{B M}(R)\right)$, we have an inclusion $\mathcal{R}_{B M}\left(M_{n}(R)\right) \subseteq M_{n}\left(\mathcal{R}_{B M}(R)\right)$. 
Let $I:=\mathcal{R}_{B M}(R)$, and $\mathcal{R}_{B M}\left(M_{n}(I)\right) \neq M_{n}(I)$. So, there exists a congruence $\rho$ on $M_{n}(I)$ such that $M_{n}(I) / \rho$ is an ideal-simple semiring with an identity $\bar{E}$ for $E \in M_{n}(I)$. By Lemma 3.14, $\bar{\rho}:=\left\{(A, B) \in M_{n}(R)^{2} \mid\right.$ $(E A E, E B E) \in \rho\}$ is a congruence on $M_{n}(R)$, and $\varphi: M_{n}(R) / \bar{\rho} \longrightarrow$ $M_{n}(I) / \rho$, given by $\bar{A} \longmapsto \overline{E A E}$, is a semiring isomorphism. It is easy to see that the relation $\theta$ defined for $a, b \in R$ as follows:

$a \theta b \Longleftrightarrow \forall i, j\left(a E_{i j} \bar{\rho} b E_{i j}\right)$, where $\left\{E_{i j}\right\}$ are the matrix units in $M_{n}(R)$,

is a congruence on $R$ and $M_{n}(R / \theta) \cong M_{n}(R) / \bar{\rho}$. Then for $\delta:=\theta \cap J^{2}$, one can readily see that $\delta$ is a congruence on $I$ and there is the injective homomorphism $\psi: I / \delta \longrightarrow R / \theta$ given by $I / \delta \ni \bar{a} \longmapsto \bar{a} \in R / \theta$ for all $a \in I$, and $\operatorname{Im}(\psi)$ is an ideal of $R / \theta$. For $M_{n}(I) / \rho \neq 0$, there exists an element $a \in I$ such that $\left(a E_{i j}, 0\right) \notin \rho$ for some $i, j$, and hence, $\left(a E_{i j}, 0\right) \notin$ $\bar{\rho}$. Therefore, $(a, 0) \notin \delta$, and $\operatorname{Im}(\psi)$ is a nonzero ideal of $R / \theta$. Since $M_{n}(I) / \rho$ is ideal-simple, so is $M_{n}(R / \theta)$, and by [15, Proposition 4.7], $R / \theta$ is also an ideal-simple semiring. Whence, $\operatorname{Im}(\psi)=R / \theta$, that is, $\psi$ is an isomorphism, and hence, $I / \delta$ is an ideal-simple semiring in a contradiction with $I=\mathcal{R}_{B M}(R)$. Thus, $\mathcal{R}_{B M}\left(M_{n}(I)\right)=M_{n}(I)$, and hence, $M_{n}(I) \subseteq$ $\mathcal{R}_{B M}\left(M_{n}(R)\right)$. Therefore, $M_{n}\left(\mathcal{R}_{B M}(R)\right)=\mathcal{R}_{B M}\left(M_{n}(R)\right)$.

(ii). First note that a simple semimodule $M \in\left|\mathcal{M}_{R}\right|$ is always unitary, that is, $1 . m=m$ for all $m \in M$. Indeed, $r(1 . m)=(r .1) m=r m$ for all $r \in R$ and consider the congruence $\beta$ on $M$ given by: $(x, y) \in \beta$ iff $r x=r y$ for all $r \in R$ and $x, y \in M$. Since $M$ is congruence-simple, $\beta=i d_{M}$ or $\beta=M^{2}$. If $\beta=M^{2}$, then $(x, 0) \in \beta$, for all $x \in M$, that is, $r x=0$ for all $r \in R$ and $x \in M$, and hence, $R M=0$. Thus, $\beta=i d_{M}$, that is, $(1 . m, m) \in \beta$, i.e., $1 . m=m$.

By [12, Theorem 5.14] and [14, Theorem 4.12], the semirings $R$ and $M_{n}(R)$ are Morita equivalent via the equivalence $F: \mathcal{M}_{R} \rightleftarrows \mathcal{M}_{M_{n}(R)}: G$ such that $F(A)=A^{n}$ and $G(B)=E_{11} B$ for all $A \in\left|\mathcal{M}_{R}\right|$ and $B \in$ $\left|\mathcal{M}_{M_{n}(R)}\right|$, where $E_{11}$ denotes the matrix unit. By Proposition 5.6, $M^{n}=$ $F(M)$ is also a simple $M_{n}(R)$-semimodule, and therefore, denoting by $\mathcal{S}_{R}$ the set of all simple $R$-semimodules, we obtain an inclusion

$$
J_{s}\left(M_{n}(R)\right) \subseteq \cap_{M \in \mathcal{S}_{R}}\left(0: M^{n}\right)_{M_{n}(R)} \subseteq M_{n}\left(J_{s}(R)\right) .
$$

Similarly, for a simple semimodule $A \in \mathcal{M}_{M_{n}(R)}$, by Proposition 5.6, $E_{11} A=G(A)$ is also a simple left $R$-semimodule, and therefore, denoting by $\mathcal{S}_{M_{n}(R)}$ the set of all simple $M_{n}(R)$-semimodules and noting the obvious inclusion $J_{s}(R) \subseteq \cap_{A \in \mathcal{S}_{M_{n}(R)}}\left(0: E_{11} A\right)_{R}$, we have the opposite inclusion

$$
M_{n}\left(J_{s}(R)\right) \subseteq M_{n}\left(\cap_{A \in \mathcal{S}_{M_{n}(R)}}\left(0: E_{11} A\right)_{R}\right) \subseteq J_{s}\left(M_{n}(R)\right) .
$$


(iii). Just using the fact that $J(R)=\left\{(0: M)_{R}|M \in| \mathcal{M}_{R} \mid\right.$ is irreducible\}, this equation can be proved similarly to (ii). Also, it was established, but by a different method, in [3, Theorem 9].

Moreover, we shall show that Theorem 5.8 can be extended to hemirings in general, but for that we first need some useful facts.

Lemma 5.9. Let $\mathbb{R}$ be a radical class of $\mathbb{H}$, and $\varrho_{\mathbb{R}}$ its radical operator having $\varrho_{\mathbb{R}}(\mathbb{N})=0$. Then, $\varrho_{\mathbb{R}}(R)=\varrho_{\mathbb{R}}\left(R^{1}\right)$ for any hemiring $R$.

Proof. It is clear that $R$ is a subtractive ideal of $R^{1}$ and $R^{1} / R \cong \mathbb{N}$. Therefore, $\varrho_{\mathbb{R}}\left(R^{1} / R\right)=\varrho_{\mathbb{R}}(\mathbb{N})=0$. By [25, Theorem 4.9], $\varrho_{\mathbb{R}}\left(R^{1}\right)=\cap\{K \in$ $\left.\mathcal{S I}\left(R^{1}\right) \mid \varrho_{\mathbb{R}}\left(R^{1} / K\right)=0\right\}$, and hence, $\varrho_{\mathbb{R}}\left(R^{1}\right) \subseteq R$. From this observation and [25, Theorem 6.2] (the $A$-D-S-property of a radical class), we have $\varrho_{\mathbb{R}}\left(R^{1}\right) \subseteq \varrho_{\mathbb{R}}(R)$. On the other hand, because of $R$ is an ideal of $R^{1}$ and [25, Theorem 6.2], $\varrho_{\mathbb{R}}(R) \subseteq \varrho_{\mathbb{R}}\left(R^{1}\right)$, and hence, $\varrho_{\mathbb{R}}\left(R^{1}\right)=\varrho_{\mathbb{R}}(R)$.

Lemma 5.10. $J(\mathbb{N})=\mathcal{R}_{B M}(\mathbb{N})=J_{s}(\mathbb{N})=0$.

Proof. For all prime numbers $p$, one can readily see that $\mathbb{N} / p \mathbb{N}=\mathbb{Z}_{p}$ is a irreducible $\mathbb{N}$-semimodule. Therefore, $J(\mathbb{N}) \subseteq \cap\left\{\left(0: \mathbb{Z}_{p}\right)_{\mathbb{N}} \mid p\right.$ is prime $\}=$ $\cap\{p \mathbb{N} \mid p$ is prime $\}=0$, and hence, $J(\mathbb{N})=0$.

(ii). $\mathcal{R}_{B M}(\mathbb{N} / p \mathbb{N})=\mathcal{R}_{B M}\left(\mathbb{Z}_{p}\right)=0$ and $p \mathbb{N}$ is a subtractive ideal of $\mathbb{N}$ for all prime numbers $p$, and hence, by [25, Theorem 4.9], $\mathcal{R}_{B M}(\mathbb{N})=\cap\{K \in$ $\left.\mathcal{S I}(\mathbb{N}) \mid \mathcal{R}_{B M}(\mathbb{N} / K)=0\right\} \subseteq \cap\{p \mathbb{N} \mid p$ is prime $\}=0$.

(iii). Obviously, $(0: \mathbf{B})_{\mathbb{N}}=0$ for the simple $\mathbb{N}$-semimodule $\mathbf{B}_{\mathbb{N}} \in\left|\mathcal{M}_{\mathbb{N}}\right|$, and therefore, $J_{s}(\mathbb{N})=0$.

Corollary 5.11. For all matrix hemirings $M_{n}(R), n \geq 1$, over a hemiring $R$, the following equations are held:

(i) $\mathcal{R}_{B M}\left(M_{n}(R)\right)=M_{n}\left(\mathcal{R}_{B M}(R)\right)$;

(ii) $J_{s}\left(M_{n}(R)\right)=M_{n}\left(J_{s}(R)\right)$;

(iii) $J\left(M_{n}(R)\right)=M_{n}(J(R))$.

Proof. (i). Applying Theorem 5.8 and Lemmas 5.9 and 5.10, one gets $M_{n}\left(\mathcal{R}_{B M}(R)\right)=M_{n}\left(\mathcal{R}_{B M}\left(R^{1}\right)\right)=\mathcal{R}_{B M}\left(M_{n}\left(R^{1}\right)\right)$. Also, since $\mathcal{R}_{B M}\left(R^{1} / R\right)$ $=\mathcal{R}_{B M}(\mathbb{N})=0$ and Lemma 5.7, one has $\mathcal{R}_{B M}\left(M_{n}\left(R^{1}\right) / M_{n}(R)\right) \cong$ $\mathcal{R}_{B M}\left(M_{n}\left(R^{1} / R\right)\right)=0$. Then, noting that $M_{n}(R)$ is a subtractive ideal of $M_{n}\left(R^{1}\right)$, since $R$ is a subtractive ideal of $R^{1}$, and applying [25, Theorem 4.9] and [25, Theorem 6.2], we have the inclusions $\mathcal{R}_{B M}\left(M_{n}\left(R^{1}\right)\right)=\cap\{K \in$ $\left.\mathcal{S I}\left(M_{n}\left(R^{1}\right)\right) \mid \mathcal{R}_{B M}\left(M_{n}\left(R^{1}\right) / K\right)=0\right\} \subseteq M_{n}(R)$ and $\mathcal{R}_{B M}\left(M_{n}\left(R^{1}\right)\right) \subseteq$ $\mathcal{R}_{B M}\left(M_{n}(R)\right)$. Furthermore, noting that $M_{n}(R)$ is an ideal of $M_{n}\left(R^{1}\right)$, since $R$ is an ideal of $R^{1}$, and applying [25, Theorem 6.2] again, we have the opposite inclusion $\mathcal{R}_{B M}\left(M_{n}\left(R^{1}\right)\right) \supseteq \mathcal{R}_{B M}\left(M_{n}(R)\right)$, and therefore, $\mathcal{R}_{B M}\left(M_{n}(R)\right)$ $=M_{n}\left(\mathcal{R}_{B M}(R)\right)$. 
Two other equations, (ii) and (iii), can be justified in the similar fashion.

As for rings (see, for example, [4, Section 4.9]), we say that a class $\delta$ of hemirings is matric-extensible, if for all natural numbers $n$, a hemiring $R \in \delta$ iff $M_{n}(R) \in \delta$.

Lemma 5.12. For any radical operator $\varrho$ on $\mathbb{H}$, hemiring $R$ and natural number $n$, there exists an ideal $I$ of $R$ such that $\varrho\left(M_{n}(R)\right)=M_{n}(I)$.

Proof. For $M_{n}(R)$ is an ideal of the semiring $M_{n}\left(R^{1}\right)$ and [25, Theorem 6.2], $\varrho\left(M_{n}(R)\right)$ is an ideal of $M_{n}\left(R^{1}\right)$. So there exists an ideal $I$ of $R^{1}$ such that $\varrho\left(M_{n}(R)\right)=M_{n}(I)$; and since $M_{n}(I) \subseteq M_{n}(R)$, one gets $I \subseteq R$.

Using this lemma, one readily obtains the following hemiring analog of [4, Theorem 4.9.3]:

Proposition 5.13. For a radical class $\gamma$ of hemirings, the following statements are equivalent:

(i) $\gamma$ is matric-extensible;

(ii) For every hemiring $R$ and natural number $n$, a matrix equation $\gamma\left(M_{n}(R)\right)=M_{n}(\gamma(R))$ is true;

(iii) The semisimple class $S_{\gamma}$ of $\gamma$ is a matric-extensible class.

Proof. (i) $\Longrightarrow$ (ii). Using Lemma 5.12 and proceeding in a similar fashion as it has been done in the proof of the implication $(1) \Longrightarrow(3)$ in $[4$, Theorem 4.9.3], one gets this implication.

(ii) $\Longrightarrow$ (iii). This can be proved in a similar way as the implication (3) $\Longrightarrow(2)$ in [4, Theorem 4.9.3].

(iii) $\Longrightarrow(\mathrm{i})$. Let $R \in \gamma$, but $M_{n}(R) \notin \gamma$. Then, $0 \neq M_{n}(R) / \gamma\left(M_{n}(R)\right) \in$ $\mathcal{S}_{\gamma}$, and from Lemma 5.12 it follows that $\gamma\left(M_{n}(R)\right)=M_{n}(I)$ for some ideal $I$ of $R$. Hence, $0 \neq M_{n}(R / I) \cong M_{n}(R) / M_{n}(I)=M_{n}(R) / \gamma\left(M_{n}(R)\right) \in \mathcal{S}_{\gamma}$. For $\mathcal{S}_{\gamma}$ is matrix-extensible, $R / I \in \mathcal{S}_{\gamma}$; on the other hand, by Theorem 2.2 (3), $R / I \in \gamma$. Thus, $R \in \gamma$ implies $M_{n}(R) \in \gamma$.

Now, let $M_{n}(R) \in \gamma$, but $R \notin \gamma$. Then, $\gamma(R) \varsubsetneqq R$ and because $\gamma(R)$ is a subtractive ideal of $R$ we have $0 \neq R / \gamma(R) \in \mathcal{S}_{\gamma}$. Hence, $M_{n}(R) / M_{n}(\gamma(R))$ $\cong M_{n}(R / \gamma(R)) \in \mathcal{S}_{\gamma}$ and $M_{n}(R / \gamma(R)) \neq 0$; however, $M_{n}(R / \gamma(R))=0$ for $M_{n}(R) \in \gamma$. Thus, $M_{n}(R) \in \gamma$ implies $R \in \gamma$.

From Corollary 5.11 and Proposition 5.13, we immediately obtain

Theorem 5.14. The radical classes of hemirings of the Jacobson radical $J$, Brown-McCoy radical $\mathcal{R}_{B M}$, and the radical $J_{s}$, are matrix-extensible classes.

Next we present some "computational" results regarding radicals of hemirings $R$ and $e R e$ for idempotents $e \in R$, which, in our view, are interesting and important on their own, and will prove to be useful in a sequel. 
Proposition 5.15. For any idempotent e of a hemiring $R$, the following statements are true:

(i) $J(e R e)=e J(R) e$;

(ii) $e R e / J(e R e) \cong \bar{e} \bar{R} \bar{e}$, where $\bar{e}$ is the image of $e$ in $\bar{R}=R / J$.

Proof. (i). For by [3, Theorem 3] $J(R)$ is a right semiregular ideal of $R$ (see, [3, Definition 3]), eae, ebe $\in J(R)$ for any $a, b \in J(R)$ and there exist elements $r_{1}, r_{2} \in J(R)$ such that

$$
e a e+r_{1}+e a e r_{1}+e b e r_{2}=e b e+r_{2}+e a e r_{2}+e b e r_{1} .
$$

So, as $e$ is an idempotent,

$$
\text { eae }+e r_{1} e+e a e . e r_{1} e+e b e . e r_{2} e=e b e+e r_{2} e+e a e . e r_{2} e+e b e . e r_{1} e .
$$

Therefore, $e J(R) e$ is a right semiregular ideal of $e R e$, and hence, by [3, theorem 3 and Definition 4], eJ(R)e $\subseteq J(e R e)$.

Now we shall show that $e J(R) e \supseteq J(e R e)$. Indeed, one can readily see that $(e R e)^{*}=\left\{r^{*} \in R^{*} \mid r \in e R e\right\}, D\left((e R e)^{*}\right)=\left\{r^{*}-s^{*} \mid r^{*}, s^{*} \in(e R e)^{*}\right\}$, $(e R e)^{*}=e^{*} R^{*} e^{*}, D\left((e R e)^{*}\right)=e^{*} D\left(R^{*}\right) e^{*}$ and, using [18, Theorem 21.10], $J\left(e^{*} D\left(R^{*}\right) e^{*}\right)=e^{*} J\left(D\left(R^{*}\right)\right) e^{*}$. Therefore,

$$
\begin{gathered}
J\left((e R e)^{*}\right)=(e R e)^{*} \cap J\left(D\left((e R e)^{*}\right)\right)= \\
=(e R e)^{*} \cap J\left(e^{*} D\left(R^{*}\right) e^{*}\right)=(e R e)^{*} \cap e^{*} J\left(D\left(R^{*}\right)\right) e^{*} \\
\subseteq e^{*}\left(R^{*} \cap J\left(D\left(R^{*}\right)\right)\right) e^{*}=e^{*} J\left(R^{*}\right) e^{*} \subseteq J\left(R^{*}\right) .
\end{gathered}
$$

So, for any $x \in J(e R e)$, we have that $x^{*} \in J\left((e R e)^{*}\right) \subseteq J\left(R^{*}\right)$, and hence, by [9, Section 4 e), p. 420], $x \in J(R)$; as $x \in J(e R e) \subseteq e R e$, we have $x=e x e \in e J(R) e$, and therefore, $J(e R e) \subseteq e J(R) e$.

(ii). Consider the natural hemiring homomorphism $f: e R e \longrightarrow \bar{e} \bar{R} \bar{e}$ given by ere $\longmapsto \bar{e} \bar{r} \bar{e}$. It is clear that $f$ induces a surjection $g: e R e / J(e R e)$ $\rightarrow \bar{e} \bar{R} \bar{e}$ that, in fact, is an isomorphism: Indeed, if $\bar{e} \bar{r} \bar{e}=\bar{e} \bar{s} \bar{e}$, then there exist elements $a, b \in J(R)$ such that $e r e+a=e s e+b$, and hence, ere + $e a e=e s e+e b e$ and, since by (i) eae, ebe $\in e J(R) e=J(e R e)$, one has ere $\equiv_{J(e R e)}$ ese.

Proposition 5.16. (i) For any $\mathcal{R}_{B M}$-semisimple semiring $R$ and an idempotent $e \in R$, the semiring eRe is also $\mathcal{R}_{B M}$-semisimple.

(ii) Let $e \in R$ be a full idempotent, i.e. Re $R=R$, in a hemiring $R$. Then, $\mathcal{R}_{B M}(e R e)=e \mathcal{R}_{B M}(R) e$ and $e R e / \mathcal{R}_{B M}(e R e) \cong \bar{e} \bar{R} \bar{e}$, where $\bar{e}$ is the image of e in $\bar{R}=R / \mathcal{R}_{B M}(R)$.

(iii) Let $e \in R$ be a full idempotent in a semiring $R$. Then, $J_{s}(e R e)=$ $e J_{s}(R) e$ and $e R e / J_{s}(e R e) \cong \bar{e} \bar{R} \bar{e}$, where $\bar{e}$ is the image of $e$ in $\bar{R}=$ $R / J_{s}(R)$. 
Proof. (i). Let $I$ be a nonzero ideal of the semiring $e$ Re, and $A:=R I R$ a nonzero ideal of $R$. For $\mathcal{R}_{B M}(R)=0$, there exists a congruence $\rho$ on $A$ such that $A / \rho$ is an ideal-simple semiring with identity $\bar{e}_{1}$. By Lemma $3.14, \bar{\rho}:=\left\{(a, b) \in R^{2} \mid\left(e_{1} a e_{1}, e_{1} b e_{1}\right) \in \rho\right\}$ is a congruence on $R$, and hence, $\theta=\bar{\rho} \cap I^{2}$ is a congruence on $I$. We shall show that $I / \theta$ is an ideal-simple semiring.

Indeed, for $e_{1} \in A$ there exist elements $r_{1}, \ldots, r_{k}, s_{1}, \ldots, s_{k} \in R$ and $a_{1}, \ldots, a_{k} \in I$ such that $e_{1}=r_{1} a_{1} s_{1}+\ldots+r_{k} a_{k} s_{k}$ with $e a_{i} e=a_{i}$ for all $a_{i}, i=1, \ldots, k$; and let $e_{2}:=e e_{1} e=e r_{1} e a_{1} e s_{1} e+\ldots+e r_{k} e a_{k} e s_{k} e \in I$. Then, $\left(a e_{2}, a\right) \in I^{2}, a e=a$, and hence, $\overline{e_{1} a e_{2} e_{1}}=\overline{e_{1} a e e_{1} e e_{1}}=\overline{e_{1} a e_{1} e e_{1}}=$ $\overline{e_{1} a e e_{1}}=\overline{e_{1} a e_{1}}$ and $\left(a e_{2}, a\right) \in \bar{\rho}$ for every element $a \in I$. Hence, $\left(a e_{2}, a\right) \in \theta$; similarly it can be shown that $\left(e_{2} a, a\right) \in \theta$, and therefore, $\bar{e}_{2}$ is the identity in the semiring $I / \theta$.

Suppose $K$ is a nonzero ideal in $I / \theta$, and let $\bar{K}:=\{x \in I \mid \bar{x} \in K\}$. For each $a \in e R e$ and $x \in \bar{K}$, we have $\bar{x} \in K, e_{2} a, a x \in I$; hence, $\overline{a x}=\overline{e_{2}} \overline{a x}=$ $\overline{e_{2} a x}=\overline{e_{2} a} \bar{x} \in K$, that is, $a x \in \bar{K}$. Similarly, we also have $x a \in \bar{K}$. So, $\bar{K}$ is an ideal of $e R e$. Then, we have $R \bar{K} R$ is an ideal of $R$ and $R \bar{K} R \subseteq A$. Consider $L:=\{\bar{y} \mid y \in R \bar{K} R\} \subseteq A / \rho$. Clearly, $L$ is an ideal of $A / \rho$. Since $K \neq 0$, there is an element $k \in I$ such that $\overline{0} \neq \bar{k} \in K$. This shows that $k \in \bar{K}$ and $\left(e_{1} k e_{1}, 0\right) \notin \rho$, and hence, $\overline{0} \neq \overline{e_{1} k e_{1}} \in L$. For $A / \rho$ is ideal-simple, $L=A / \rho$; hence, $\bar{e}_{1} \in L$, i.e., there exist elements $r_{1}, \ldots, r_{l}, s_{1}, \ldots, s_{l} \in R$ and $x_{1}, \ldots, x_{l} \in \bar{K}$ such that $\overline{e_{1}}=\overline{r_{1} x_{1} s_{1}+\ldots+r_{l} x_{l} s_{l}}$. As $x_{i} \in \bar{K}$, we have $e x_{i} e=x_{i}$ for all $i=1, \ldots l$. Then, as $\bar{K}$ is an ideal of $e$ Re, for all $i=1, \ldots l$, we have $e r_{i} x_{i} s_{i} e=e r_{i} e x_{i} e s_{i} e \in \bar{K}$ and $e r_{1} x_{1} s_{1} e+\ldots+e r_{l} x_{l} s_{l} e \in \bar{K}$. Whence, $\bar{e}_{2}=\overline{e e_{1} e}=\overline{e r_{1} x_{1} s_{1} e+\ldots+e r_{l} x_{l} s_{l} e} \in K$, and hence, $K=I / \theta$. Thus, $I / \theta$ is an ideal-simple semiring, and therefore, $\mathcal{R}_{B M}(e R e)=0$.

(ii). As $\mathcal{R}_{B M}(R)$ is a subtractive ideal of $R$, the ideal $e \mathcal{R}_{B M}(R) e$ of $e R e$ is also subtractive. Similarly to the proof of Proposition 5.15 (ii), it can be shown that $e R e / e \mathcal{R}_{B M}(R) e \cong \bar{e} \bar{R} \bar{e}$, where $\bar{e}$ is the image of $e$ in $\bar{R}=R / \mathcal{R}_{B M}(R)$.

From $\mathcal{R}_{B M}\left(R / \mathcal{R}_{B M}(R)\right)=0$ and (i), it follows that $\mathcal{R}_{B M}\left(e R e / e \mathcal{R}_{B M}(R) e\right)=0$ and by [25, Theorem 4.9] we have the inclusion $\mathcal{R}_{B M}(e R e)=\cap\left\{K \in \mathcal{S I}(e R e) \mid \mathcal{R}_{B M}(e R e / K)=0\right\} \subseteq e \mathcal{R}_{B M}(R) e$. And we need only to show that the opposite inclusion $e \mathcal{R}_{B M}(R) e \subseteq \mathcal{R}_{B M}(e R e)$ also takes place.

So, suppose $e \mathcal{R}_{B M}(R) e \nsubseteq \mathcal{R}_{B M}(e R e)$. Then there exists a congruence $\rho$ on $e \mathcal{R}_{B M}(R) e$ such that $e \mathcal{R}_{B M}(R) e / \rho$ is an ideal-simple semiring with identity $\overline{e_{1}}$ with $e_{1} \in e \mathcal{R}_{B M}(R) e$. Similarly to the proof in (i) and applying Lemma 3.14 and [16, Propostion 5.3], we have that

$$
\bar{\rho}=\left\{(a, b) \in(e R e)^{2} \mid\left(e_{1} a e_{1}, e_{1} b e_{1}\right) \in \rho\right\}
$$


is a congruence on $e R e$ and the relation $\theta$ on $R$, defined for all $a, b \in R$ by

$$
(a, b) \in \theta \Longleftrightarrow \forall r, s \in R:(\text { erase }, \text { erbse }) \in \bar{\rho},
$$

is a congruence on $R$. Now we shall show that for the congruence $\delta:=\theta \cap$ $\mathcal{R}_{B M}(R)^{2}$ on $\mathcal{R}_{B M}(R)$, the hemiring $\mathcal{R}_{B M}(R) / \delta$ is, in fact, an ideal-simple semiring. For $e$ is a full idempotent, $1=x_{1} e y_{1}+\ldots+x_{n} e y_{n}$ for some $x_{i}, y_{i} \in$ $R, i=1, \ldots, n$, and $e e_{1}=e_{1}=e_{1} e \in e \mathcal{R}_{B M}(R) e \subseteq \mathcal{R}_{B M}(R)$. For any $x \in$ $\mathcal{R}_{B M}(R)$ and $r, s \in R$, we have $e_{1}$ er $_{i} e_{1} y_{i} x s e e_{1}=e e_{1}$ er $x_{i} e . e_{1}$.ey $y_{i} x$ see $_{1} e$ and $e e_{1}$ erx $_{i} e$, ey $_{i} x_{\text {see }} e \in e \mathcal{R}_{B M}(R) e$. Whence, $\left(e_{1}\right.$ erx $_{i} e_{1} y_{i} x_{\text {see }}, e_{1}$ erx $_{i}$ ey $y_{i}$ see $\left._{1}\right)$ $\in \rho$ for all $i=1, \ldots, n$, and $\left(e_{1} \operatorname{er}\left(\sum_{i=1}^{n} x_{i} e_{1} y_{i}\right)\right.$ xsee $_{1}, e_{1}$ erxsee $\left._{1}\right)=$ $\sum_{i=1}^{n}\left(e_{1}\right.$ er $_{i} e_{1} y_{i}$ xsee $_{1}, e_{1}$ er $_{i}$ ey $_{i}$ xsee $\left._{1}\right) \in \rho$, and hence, $\left(\left(\sum_{i=1}^{n} x_{i} e_{1} y_{i}\right) x, x\right) \in$ $\theta$. Furthermore, for $e_{2}:=\sum_{i=1}^{n} x_{i} e_{1} y_{i} \in \mathcal{R}_{B M}(R)$ and any $x \in \mathcal{R}_{B M}(R)$, we have $\left(e_{2} x, x\right) \in \mathcal{R}_{B M}(R)^{2}$, and hence, $\left(e_{2} x, x\right) \in \delta$; similarly, $\left(x e_{2}, x\right) \in \delta$ too. Thus, $\mathcal{R}_{B M}(R) / \delta$ is a semiring with identity $\overline{e_{2}}$.

Let $I$ be a nonzero ideal of $\mathcal{R}_{B M}(R) / \delta$, and $\bar{I}:=\left\{x \in \mathcal{R}_{B M}(R) \mid \bar{x} \in I\right\}$. For each $r \in R$ and $x \in \bar{I}$, we have $e_{2} r, r x \in \mathcal{R}_{B M}(R)$, and hence, $\overline{r x}=$ $\overline{e_{2}} \cdot \overline{r x}=\overline{e_{2} r} \cdot \bar{x} \in I$ and $r x \in \bar{I}$. Similarly, one also gets $x r \in \bar{I}$. Therefore, $\bar{I}$ is an ideal of $R$. It is clear that $K:=\{\bar{x} \mid x \in e \bar{I} e\} \subseteq e \mathcal{R}_{B M}(R) e / \rho$ is an ideal of $e \mathcal{R}_{B M}(R) e / \rho$. For $I \neq 0$, there exists an element $a \in \mathcal{R}_{B M}(R)$ such that $0 \neq \bar{a} \in I$. It shows that $a \in \bar{I}$ and $(a, 0) \notin \delta$, that is, there exist $r, s \in R$ such that (erase, 0$) \notin \bar{\rho}$, i.e., $\left(e_{1}\right.$ erasee $\left._{1}, 0\right) \notin \rho$, and $K \neq 0$ because $e_{1}$ erasee $_{1} \in K$. For $e \mathcal{R}_{B M}(R) e / \rho$ is ideal-simple, $K=e \mathcal{R}_{B M}(R) e / \rho$. Then, $\overline{e_{1}} \in K$, that is, $e_{1} \in e \bar{I} e$. As $\bar{I}$ is an ideal of $R$, we have $e \bar{I} e \subseteq \bar{I}$, and hence, $e_{1} \in \bar{I}$ and $e_{2}=\sum_{i=1}^{n} x_{i} e_{1} y_{i} \in \bar{I}$. Whence, $\overline{e_{2}} \in I$, and hence, $I=\mathcal{R}_{B M}(R) / \delta$. Therefore, $\mathcal{R}_{B M}(R) / \delta$ is an ideal-simple semiring; however, $\mathcal{R}_{B M}(R)$ has no nonzero semiring images. Thus, $e \mathcal{R}_{B M}(R) e \subseteq \mathcal{R}_{B M}(e R e)$.

(iii). Similarly to the proof of Proposition 5.1, we readily have that $P=R e$ is a progenerator of ${ }_{R} \mathcal{M}$ and $P^{*}=\operatorname{Hom}_{R}\left({ }_{R} P,{ }_{R} R\right) \cong e R, S=$ $\operatorname{End}_{R}\left({ }_{R} P\right) \cong e R e$, as well as there exist the isomorphisms $\alpha: P \otimes_{e R e} P^{*}=$ $R e \otimes_{e R e} e R \longrightarrow R$ and $\beta: P^{*} \otimes_{R} P=e R \otimes_{R} R e \longrightarrow e R e$ such that $\alpha\left(r e \otimes e r^{\prime}\right)=r e r^{\prime}$ and $\beta\left(e r \otimes r^{\prime} e\right)=e r r^{\prime} e$. Also, similarly to as it was done in the proof of [14, Corollary 4.4], we get the inverse category equivalences: $F:{ }_{R} \mathcal{M} \rightleftarrows{ }_{e R e} \mathcal{M}: G$ given by $F(A)=P^{*} \otimes_{R} A=e R \otimes_{R} A \cong e A$ and $G(B)=P \otimes_{e R e} B=\operatorname{Re} \otimes_{e R e} B$. Therefore, by Proposition 5.6, the functors $F$ and $G$ establish the equivalences between the categories of simple semimodules of the categories ${ }_{R} \mathcal{M}$ and $e R e \mathcal{M}$, respectively.

Let $\mathcal{S}_{R}$ stay for the set of all simple left $R$-semimodules. Then, $J_{s}(R)=$ $\cap_{M \in \mathcal{S}_{R}}(0: M)_{R}$ and $J_{s}(e R e)=\cap_{M \in \mathcal{S}_{R}}(0: F(M))_{e R e}=\cap_{M \in \mathcal{S}_{R}}(0: e M)_{e R e}$. And we shall prove that $e J_{s}(R) e=\cap_{M \in \mathcal{S}_{R}}(0: e M)_{e R e}$. Indeed, if ere $\in$ $\cap_{M \in \mathcal{S}_{R}}(0: e M)_{e R e}$, then ere $M=$ ere.e $M=0$ for all $M \in \mathcal{S}_{R}$, and therefore, ere $\in J_{s}(R)$ and ere $\in e J_{s}(R) e$, and hence, $\cap_{M \in \mathcal{S}_{R}}(0: e M)_{e R e} \subseteq e J_{s}(R) e$. 
Conversely, if $r \in J_{s}(R)$, then $r M=0$ for all $M \in \mathcal{S}_{R}$; so, er $M=0$ for all $M \in \mathcal{S}_{R}$. As $e M \subseteq M$, we have ere.e $M=$ ere $M$ for all $M \in \mathcal{S}_{R}$. Whence, ere $\in \cap_{M \in \mathcal{S}_{R}}(0: e M)_{e R e}$, and hence, $e J_{s}(R) e \subseteq \cap_{M \in \mathcal{S}_{R}}(0: e M)_{e R e}$.

The rest can be proved in the similar to the proof of Proposition 5.15 (ii) way.

Our next result shows that the $J$-semisimplicity, $J_{s}$-semisimplicity, and $\mathcal{R}_{B M}$-semisimplicity of semirings are Morita invariant properties, namely:

Theorem 5.17. For Morita equivalent semirings $R$ and $S$, the followings statements are true:

(i) $J(R)=0$ iff $J(S)=0$, and the semirings $R / J(R)$ and $S / J(S)$ are Morita equivalent;

(ii) $\mathcal{R}_{B M}(R)=0$ iff $\mathcal{R}_{B M}(S)=0$, and the semirings $R / \mathcal{R}_{B M}(R)$ and $S / \mathcal{R}_{B M}(S)$ are Morita equivalent;

(iii) $J_{s}(R)=0$ iff $J_{s}(S)=0$, and the semirings $R / J_{s}(R)$ and $S / J_{s}(S)$ are Morita equivalent.

Proof. (i). The assertion that $J(R)=0$ iff $J(S)=0$ follows right away from Proposition 5.1 and Theorem 5.8 or Corollary 5.11.

Next, by Proposition 5.1, $S \cong e M_{n}(R) e$ for some idempotent $e \in M_{n}(R)$ such that $M_{n}(R) e M_{n}(R)=M_{n}(R)$. Then, it is clear that $M_{n}(R /(J(R)) \cong$ $M_{n}(R) / M_{n}(J(R))$. From these observations, Proposition 5.15 and Theorem 5.8 , we have that $S / J(S) \cong \bar{e} M_{n}(R / J(R)) \bar{e}$ and $M_{n}(R / J(R)) \bar{e} M_{n}(R / J(R))$ $=M_{n}(R / J(R))$, and, using Proposition 5.1 again, we obtain that $R / J(R)$ and $S / J(S)$ are Morita equivalent.

Just using Proposition 5.16, (ii) and (iii) are proved in the same fashion as (i).

Two hemirings $R$ and $S$, we say, are $D$-Morita equivalent if their Dorroh extensions $R^{1}$ and $S^{1}$, respectively, are Morita equivalent semirings. Since, by [14, Corollary 4.4], the Morita equivalence relation on the category of semirings is an equivalence relation, the D-Morita equivalence relation for hemirings is also an equivalence relation on the category of hemirings.

Using Lemmas 5.9, 5.10 and Theorem 5.17, we conclude the paper with the following result:

Corollary 5.18. For D-Morita equivalent hemirings $R$ and $S$, the followings statements are true:

(i) $J(R)=0$ iff $J(S)=0$;

(ii) $\mathcal{R}_{B M}(R)=0$ iff $\mathcal{R}_{B M}(S)=0$;

(iii) $J_{s}(R)=0$ iff $J_{s}(S)=0$. 


\section{References}

[1] Bashir, R. El , Hurt, J., Jančařík, A., Kepka, T. (2001). Simple Commutative Semirings. J. Algebra 236:277-306.

[2] Bashir, R. El , Kepka, T. (2007). Congruence-Simple Semirings. Semigroup Forum 75:588-608.

[3] Bourne, S. (1951). The Jacobson radical of a semiring. Proc. Nat. Acad. Sci. U.S.A. 37:163-170.

[4] Gardner, B. J., Wiegandt, R. (2004). Radical theory of rings. New York-Basel: Marcel Dekker.

[5] Golan, J. S. (1999). Semirings and their Applications. DordrechtBoston-London: Kluwer Academic Publishers.

[6] Hebisch, U., Weinert, H. J. (1997). Radical theory for semirings. Quaestiones Mathematicae 20:647-661.

[7] Hebisch, U., Weinert, H. J. (2001). On the interrelation between radical theories for semirings and rings. Comm. Algebra 29:109-129.

[8] Hebisch, U., Weinert, H. J. (2002). Semisimple classes of semirings. Algebra Colloquium 9:177-196.

[9] Ilzuka, K. (1959). On the Jacobson radical of a semiring. Tohuku Math. J. 11:409-421.

[10] Izhakian, Z., Rhodes, J., Steinberg, B. (2011). Representation theory of finite semigroups over semirings. Journal of Algebra 336:139-157.

[11] Ježek, J., Kepka, T., Maróti, M. (2009). The endomorphism semiring of a semilattice. Semigroup Forum 78:21-26.

[12] Katsov, Y. (2004). Toward homological characterization of semirings: Serre's conjecture and Bass's perfectness in a semiring context, Algebra Universalis 52:197-214.

[13] Katsov, Y., Nam, T. G., Tuyen, N. X. (2009). On subtractive semisimple semirings. Algebra Colloquium 16:415-426.

[14] Katsov, Y., Nam, T. G. (2011). Morita Equivalence and Homological Characterization of Semirings. J. Algebra Appl. 10:445 - 473. 
[15] Katsov, Y., Nam, T. G., Tuyen, N. X. (2011). More on Subtractive Semirings: Simpleness, Perfectness and Related Problems. Comm. Algebra 39:4342 - 4356.

[16] Katsov, Y., Nam, T. G., Zumbrägel, J. (2011). On Simpleness of Semirings and Complete Semirings (arXiv:1105.5591v1).

[17] Lam, T. Y. (1999). Lectures on Modules and Rings. New York-Berlin: Springer-Verlag.

[18] Lam, T. Y. (2001). A first course in noncommutative rings, 2nd Ed. New York-Berlin: Springer-Verlag.

[19] LaTorre, D. R. (1967 ). A note on the Jacobson radical of a hemiring. Publ. Math. Debrecen 14:9-13.

[20] LaTorre, D. R. (1967). The Brown-McCoy radicals of a hemiring. Publ. Math. Debrecen 14:15-28.

[21] Latorre, D. R. (1971). Modular congruence and the Brown-McCoy radical for semigroups. Proceedings of The American Mathematical Society 29: $427-433$.

[22] Mac Lane, S. (1998). Categories for the Working Mathematician, 2nd Ed. New York-Berlin: Springer-Verlag.

[23] Mitchell, S. S., Fenoglio, P.B. (1988). Congruence-free commutative semirings. Semigroup Forum 31:79-91.

[24] Monico, C. (2004). On finite congruence-simple semirings. J. Algebra 271:846-854.

[25] Morak, B. (1999). On the radical theory for semirings. Beitrage zur Algebra und Geometrie 40:533-549.

[26] Olson, D. M., Jenkins, T. L. (1983). Radical theory for hemirings. J. Natur. Sci. Math. 23:23-32.

[27] Olson, D. M., Nance, A. C. (1989). A note on radical for hemirings. Quaestiones Mathematicae 12:307-314.

[28] Olson, D. M., Heyman, G. A. P., LeRoux, L. H. (1992). Weakly special classes of hemirings. Quaestiones Mathematicae 15:119-126.

[29] Olson, D. M., LeRoux, L. H., Heyman, G. A. P. (1994). Three special classes for hemirings. Quaestiones Mathematicae 17:205-215. 
[30] Weinert, H. J., Weigandt, R. (1992). A Kurosh-Amitsur radical theory for proper semifields. Comm. Algebra 20:2419-2458.

[31] Weinert, H. J., Weigandt, R. (2003). A new Kurosh-Amitsur radical theory for proper semifields I. Mathematica Pannonica 14:3-28.

[32] Weinert, H. J., Weigandt, R. (2003). A new Kurosh-Amitsur radical theory for proper semifields II, Mathematica Pannonica 14:149-164.

[33] Zumbrägel, J. (2008). Classification of finite congruence-simple semirings with zero. J. Algebra Appl. 7:363-377. 